\title{
Convexity and the quantum many-body problem
}

\author{
P Chau Huu-Tai ${ }^{1}$ and $\mathrm{P}$ Van Isacker ${ }^{2}$ \\ ${ }^{1}$ CEA/DAM/DIF, F-91297 Arpajon, France \\ ${ }^{2}$ Grand Accélérateur National d'Ions Lourds (GANIL), CEA/DSM-CNRS/IN2P3, \\ Bd Henri Becquerel, F-14076 Caen, France \\ E-mail: huu-tai.chau@cea.fr
}

\begin{abstract}
We recall some properties of convex functions and, in particular, of the sum of the largest eigenvalues of a Hermitian matrix. From these properties a new estimate of an arbitrary eigenvalue of a sum of Hermitian matrices is derived, which in turn is used to compute an approximate associated spectral projector. These estimates are applied for the first time to explain generic spectral features of quantum systems. As an application of the formalism, we explain the preponderance of certain groundstate angular momenta as observed in the vibron model with random interactions. We show that the evolution of eigenstates can be predicted from the knowledge of a limited number of spectra and investigate the effect of a three-body interaction in the vibron model on eigenenergies and eigenvectors.
\end{abstract}

\section{Introduction}

In non-relativistic quantum mechanics the properties of a many-particle system are obtained by solving the Schrödinger equation. Usually this equation reduces to the diagonalization of a Hamiltonian matrix which is Hermitian and the elements of which depend on the interaction between the particles. It is often assumed that this interaction is of finite rank (one-body, two-body,...), and the Hamiltonian matrix can then be rewritten as a sum of a set of given Hermitian matrices. It is therefore useful to study the generic properties of the eigenvalues of sums of Hermitian matrices. Since the pioneering work of Weyl [1], many inequalities (e.g., by Lidskii [2]) have been derived for eigenvalues of sums of Hermitian matrices; a review can be found in, e.g., Bhatia's book [3]. More recently, differential properties of the eigenvalues of symmetric and Hermitian matrices have been investigated within the non-smooth analysis framework [4].

In this article we deduce a set of inequalities from the convexity property of the sum of the largest eigenvalues of a Hermitian matrix. From these inequalities, estimates are derived for such sums as well as for the eigenvalues of a sum of Hermitian matrices. Moreover, since expressions of spectral projectors are known in terms of the eigenvalues of a Hermitian matrix, the derived estimates of the eigenvalues can be used to obtain approximate spectral projectors. 
Formal properties of convex functions are given in section 2, which begins with a summary of known definitions and properties, leading to a new and generic estimate of an arbitrary eigenvalue of a sum of Hermitian matrices. Given that almost any nonrelativistic theory of interacting particles can be reduced to the diagonalization of sums of Hermitian matrices, the formal results of section 2 have immediate repercussions in quantum mechanics, as explained in section 3. It has to be noted that, since convexity is a global feature, the derived estimates are not limited to small perturbations. Moreover, they do not require differentiability of eigenvalues which in general is not satisfied in the case of degenerate eigenvalues. Hence, the eigenvalue estimates given in this paper are able to cope with level crossings.

To illustrate the use of the derived estimates, we apply them in section 4 to predict some spectral properties of the vibron model, which was proposed by Iachello and Levine [5] in the study of molecules. Since the inequalities do not depend on specific parameters but are generally valid, they can be used to shed new light on some known (but nevertheless startling) results concerning the ground-state angular momentum obtained in the vibron model with random interactions. We also study the effect of a three-body interaction on the spectrum of the vibron model. Finally, conclusions are drawn in section 5 .

\section{Convexity}

\subsection{Convex functions}

We introduce some definitions and notations and recall some theorems about convexity. More details and proofs can be found in e.g. $[6,7,8]$.

Let $\mathcal{C}$ be a non-empty convex set in $\mathbb{R}$. A function $f: \mathcal{C} \rightarrow \mathbb{R}$ is convex over $\mathcal{C}$ if

$$
\forall(x, y) \in \mathcal{C} \times \mathcal{C}, \forall \alpha \in[0,1]: f(\alpha x+(1-\alpha) y) \leq \alpha f(x)+(1-\alpha) f(y) .
$$

Example 1. Let us consider the following function $g$ defined on $[0,1]$ by

$$
\left\{\begin{array}{l}
x \leq x_{0}, g(x)=3\left(x-u_{0}\right)^{2} \\
x_{0} \leq x, g(x)=x^{5}
\end{array}\right.
$$

with $x_{0}=3 / 5$ and $u_{0}=x_{0}+x_{0}^{5 / 2} / \sqrt{3} . g$ is a convex function whose graph is plotted in figure 1(a).

A function $f: \Omega \rightarrow \mathbb{R}$, where $\Omega$ is an open set in $\mathbb{R}$, has a right (left) derivative $f_{+}^{\prime}\left(x_{0}\right)\left(f_{-}^{\prime}\left(x_{0}\right)\right)$ at $x_{0}$ in $\Omega$, if the limit

$$
\lim _{h \rightarrow 0^{ \pm}} \frac{f\left(x_{0}+h\right)-f\left(x_{0}\right)}{h} \equiv f_{ \pm}^{\prime}\left(x_{0}\right)
$$

exists.

If $\mathcal{C}$ is a convex subset of $\Omega$ and if the function $f$ is convex, then $f$ has a right and a left derivative for all $x_{0} \in \mathcal{C}$ and the following inequalities hold:

$$
\begin{aligned}
& \forall x \in \mathcal{C}, x \leq x_{0}: f(x) \geq f\left(x_{0}\right)+f_{-}^{\prime}\left(x_{0}\right)\left(x-x_{0}\right), \\
& \forall x \in \mathcal{C}, x \geq x_{0}: f(x) \geq f\left(x_{0}\right)+f_{+}^{\prime}\left(x_{0}\right)\left(x-x_{0}\right) .
\end{aligned}
$$


Under the same assumptions for $f$ and $\mathcal{C}$, we have that

$$
\forall x_{0} \in \mathcal{C}: f_{-}^{\prime}\left(x_{0}\right) \leq f_{+}^{\prime}\left(x_{0}\right)
$$

and therefore we can choose $K\left(x_{0}\right)$ such that $f_{-}^{\prime}\left(x_{0}\right) \leq K\left(x_{0}\right) \leq f_{+}^{\prime}\left(x_{0}\right)$. The inequalities (2) then imply

$$
\forall\left(x, x_{0}\right) \in \mathcal{C} \times \mathcal{C}: f(x) \geq f\left(x_{0}\right)+K\left(x_{0}\right)\left(x-x_{0}\right) .
$$

$K\left(x_{0}\right)$ is called a subgradient of $f$ at $x_{0}$ and the set of subgradients of $f$ at $x_{0}$ is called the subdifferential of $f$ at $x_{0}$ and it is denoted by $\partial f\left(x_{0}\right)$.

Example 2. The function $g$ defined by equation (1) has a derivative for all $x \neq x_{0}$. At $x_{0}, g$ has left and right derivatives. The inequality (3) is illustrated in figure 1(b) where the straigth lines are lower limits of the function.

On the basis of the latter property, we can propose an estimate of a convex function if some of its values are known.

Theorem 1. Let $\Omega$ be an open set in $\mathbb{R}$ and $f$ be a convex function over $\Omega \supset[a, b]$ with $a<b$. Assuming that $f \in \mathscr{C}^{1}([a, b])$, i.e. $f$ is differentiable over $[a, b]$ with a continuous derivative, we define the function $\tilde{f}$ as

$$
\begin{aligned}
\forall c \in[a, b]: \tilde{f}(c) & =\frac{1}{2} \max \left(f(a)+f^{\prime}(a)(c-a), f(b)+f^{\prime}(b)(c-b)\right) \\
& +\frac{1}{2}((1-\alpha) f(a)+\alpha f(b)),
\end{aligned}
$$

where $\alpha=(c-a) /(b-a)$ is the barycentric coordinate of $c$ in $[a, b]$.

Then the function $\tilde{f}$ is an estimate of $f$, which satisfies

$$
|f(c)-\tilde{f}(c)| \leq \frac{1}{2} \max (\alpha, 1-\alpha)(b-a)\left(f^{\prime}(b)-f^{\prime}(a)\right) .
$$

Proof. (i) If $f^{\prime}(a)=f^{\prime}(b)$ : Since $f$ is convex, its derivative $f^{\prime}$ is a monotonously increasing function and, therefore, $\forall c \in[a, b]: f^{\prime}(c)=f^{\prime}(a)$, which means that $f$ is an affine function and $\tilde{f}(c)=f(c)$.

(ii) If $f^{\prime}(a) \neq f^{\prime}(b)$ : We introduce $\bar{c}$ such that $f(a)+f^{\prime}(a)(\bar{c}-a)=f(b)+f^{\prime}(b)(\bar{c}-b)$. The function $\tilde{f}$ alternatively can be defined as

$$
\begin{aligned}
& \forall c \leq \bar{c}: \tilde{f}(c)=\frac{1}{2}\left(f(a)+f^{\prime}(a)(c-a)+(1-\alpha) f(a)+\alpha f(b)\right), \\
& \forall c \geq \bar{c}: \tilde{f}(c)=\frac{1}{2}\left(f(b)+f^{\prime}(b)(c-b)+(1-\alpha) f(a)+\alpha f(b)\right),
\end{aligned}
$$

which allows for a separate treatment of the cases $c \leq \bar{c}$ and $c \geq \bar{c}$. We note that the following implication is generally valid:

$$
\left.\forall A, B, C, D \in \mathbb{R}: \begin{array}{c}
A \leq C \leq B \\
D=\frac{1}{2}(A+B)
\end{array}\right\} \Rightarrow|D-C| \leq \frac{1}{2}(B-A) .
$$

The application of the property (2) for $x_{0}=a$ leads to the inequality

$$
\forall c \in] a, b\left[: f(c) \geq f(a)+\alpha(b-a) f^{\prime}(a) .\right.
$$



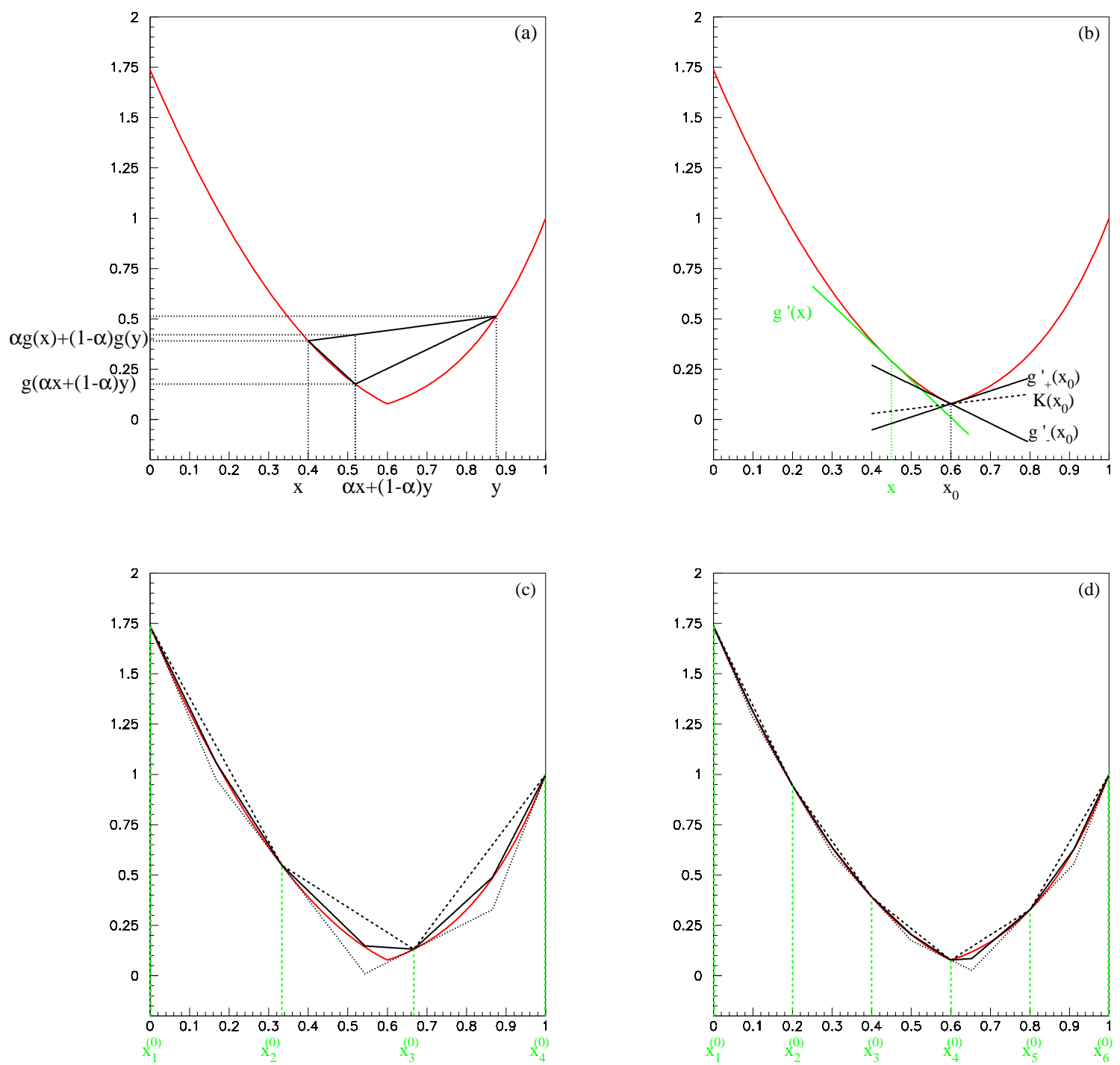

Figure 1. (a) Graph of the convex function $g$ defined by equation (1). (b) Illustration of the inequality (3) applied to $g$. (c) Estimate of $g$ according to theorem 2 using 4 values of $g$ and of its subgradients. (d) Same as (c) for 6 values.

The preliminary inequality (6) with $A=f(a)+f^{\prime}(a)(c-a), B=(1-\alpha) f(a)+\alpha f(b)$, $C=f(c)$ and $D=\tilde{f}(c)$ therefore gives for $c \leq \bar{c}$

$$
|f(c)-\tilde{f}(c)| \leq \frac{1}{2}\left((1-\alpha) f(a)+\alpha f(b)-f(a)-f^{\prime}(a)(c-a)\right),
$$

or

$$
|f(c)-\tilde{f}(c)| \leq \frac{1}{2}(c-a)\left(\frac{f(b)-f(a)}{b-a}-f^{\prime}(a)\right) .
$$

Since $f \in \mathscr{C}^{1}([a, b])$, the mean-value theorem is valid and implies that

$$
\exists d \in] a, b\left[: f^{\prime}(d)=\frac{f(b)-f(a)}{b-a} .\right.
$$


With the help of this result, the last inequality can be rewritten as

$$
|f(c)-\tilde{f}(c)| \leq \frac{1}{2}(c-a)\left(f^{\prime}(d)-f^{\prime}(a)\right) .
$$

Due to the convexity of $f$, its derivative $f^{\prime}$ is a monotonously increasing function, and therefore

$$
|f(c)-\tilde{f}(c)| \leq \frac{1}{2}(c-a)\left(f^{\prime}(b)-f^{\prime}(a)\right)=\frac{1}{2} \alpha(b-a)\left(f^{\prime}(b)-f^{\prime}(a)\right) .
$$

The case $c \geq \bar{c}$ can be treated similarly and leads to

$$
|f(c)-\tilde{f}(c)| \leq \frac{1}{2}(1-\alpha)(b-a)\left(f^{\prime}(b)-f^{\prime}(a)\right) .
$$

Combination of the two cases $c \leq \bar{c}$ and $c \geq \bar{c}$ leads to the theorem.

Theorem 2. Let $f$ be a convex function over $\Omega \supset[a, b]$ with $a<b$. We define $a$ function, $K$, by

$$
\begin{aligned}
K: & {[a, b] \rightarrow \mathbb{R}, } \\
& x_{0} \mapsto K\left(x_{0}\right) \in \partial f\left(x_{0}\right),
\end{aligned}
$$

so that this function satisfies

$$
\forall\left(x, x_{0}\right) \in[a, b] \times[a, b]: f(x) \geq f\left(x_{0}\right)+K\left(x_{0}\right)\left(x-x_{0}\right) .
$$

We define the function $\tilde{f}$ as

$$
\begin{aligned}
\forall c \in[a, b]: \tilde{f}(c) & =\frac{1}{2}(\max (f(a)+K(a)(c-a), f(b)+K(b)(c-b))) \\
& +\frac{1}{2}((1-\alpha) f(a)+\alpha f(b)),
\end{aligned}
$$

with $\alpha=(c-a) /(b-a)$.

Then the function $\tilde{f}$ is an estimate of $f$, which satisfies

$$
|f(c)-\tilde{f}(c)| \leq \frac{1}{2} \max (\alpha, 1-\alpha)(b-a)(K(b)-K(a)) .
$$

Proof. The derivation of this result relies on two key arguments, namely the mean-value theorem and the monotonicity property of the subdifferential which are both true for any convex function even if this function is not differentiable (see e.g. [8], theorem 2.3.3, page 179 and proposition 6.1.1, page 199, respectively).

(i) If $K(a)=K(b)$ : The monotonicity property of the subdifferential implies that

$$
\forall c \in[a, b]: K(c)=K(a) .
$$

Moreover

$$
\forall s \in \partial f(c): K(a) \leq s \leq K(b) \Rightarrow s=K(a)=K(b) .
$$

Using the mean-value theorem, it is deduced that

$$
\forall c \in] a, b[: f(c)-f(a)=K(a)(c-a),
$$

which means that $f$ is an affine function and that $\tilde{f}=f$. 
(ii) If $K(a) \neq K(b)$ : We introduce $\bar{c}$ such that $f(a)+K(a)(\bar{c}-a)=f(b)+K(b)(\bar{c}-b)$. The function $\tilde{f}$ alternatively can be defined as

$$
\begin{aligned}
& \forall c \leq \bar{c}: \tilde{f}(c)=\frac{1}{2}(f(a)+K(a)(c-a)+(1-\alpha) f(a)+\alpha f(b)), \\
& \forall c \geq \bar{c}: \tilde{f}(c)=\frac{1}{2}(f(b)+K(b)(c-b)+(1-\alpha) f(a)+\alpha f(b)),
\end{aligned}
$$

which allows for a separate treatment of the cases $c \leq \bar{c}$ and $c \geq \bar{c}$. The application of the definition of the convexity and of the property (2) for $x_{0}=a$ leads to the inequalities

$$
\forall c \leq \bar{c}: f(a)+K(a)(c-a) \leq f(c) \leq(1-\alpha) f(a)+\alpha f(b) .
$$

The preliminary result (6) with $A=f(a)+K(a)(c-a), B=(1-\alpha) f(a)+\alpha f(b)$, $C=f(c)$ and $D=\tilde{f}(c)$ therefore gives for $c \leq \bar{c}$

$$
|f(c)-\tilde{f}(c)| \leq \frac{1}{2}((1-\alpha) f(a)+\alpha f(b)-f(a)-K(a)(c-a)),
$$

or

$$
|f(c)-\tilde{f}(c)| \leq \frac{1}{2}(c-a)\left(\frac{f(b)-f(a)}{b-a}-K(a)\right) .
$$

Because of the mean-value theorem which states that

$$
\exists d \in] a, b\left[: \frac{f(b)-f(a)}{b-a} \in \partial f(d),\right.
$$

and because of the monotonicity property of the subdifferential which implies

$$
\frac{f(b)-f(a)}{b-a} \leq K(b)
$$

we obtain that

$$
|f(c)-\tilde{f}(c)| \leq \frac{1}{2}(c-a)(K(b)-K(a))=\frac{1}{2} \alpha(b-a)(K(b)-K(a)) .
$$

The case $c \geq \bar{c}$ can be treated similarly and leads to

$$
|f(c)-\tilde{f}(c)| \leq \frac{1}{2}(1-\alpha)(b-a)(K(b)-K(a)) .
$$

Combination of the two cases $c \leq \bar{c}$ and $c \geq \bar{c}$ leads to the theorem.

Example 3. Theorem 2 is illustrated in figure 1(c) for the function g where it is assumed that 4 values of $g$ and 4 of its subgradients are known at $x_{i}^{(0)}$ with $i=1, \ldots, 4$. The dashed and the dotted lines represent $\max (f(a)+K(a) c-a), f(b)+K(b)(c-b))$ and $(1-\alpha) f(a)+\alpha f(b)$, respectively, with $a=x_{i}^{(0)}$ and $b=x_{i+1}^{(0)}$. The full black lines represent the estimate of $g$ as defined in theorem 2. Figure 1(d) illustrates the same for 6 values. 


\subsection{Extension to higher dimensions}

The definitions and theorems of the previous subsection can be extended to higher dimensions by introducing directional derivatives. First, we introduce the notion of a convex set in $\mathbb{R}^{p}$ and a convex function over that set. Assume $k$ points $\left\{t_{1}, \ldots, t_{k}\right\}$ with $t_{i} \in \mathbb{R}^{p}$. This ensemble defines a convex set, denoted here by $\mathcal{C}$, which contains all points $t$ that satisfy

$$
\exists \alpha_{i} \in[0,1]: t=\sum_{i=1}^{k} \alpha_{i} t_{i}, \quad \sum_{i=1}^{k} \alpha_{i}=1
$$

In the following $t_{i}, \alpha_{i}$ and $t$ are taken according to the above definitions. A function $f: \mathcal{C} \rightarrow \mathbb{R}$ is convex over $\mathcal{C}$ if

$$
\forall t \in \mathcal{C}: f(t) \leq \sum_{i=1}^{k} \alpha_{i} f\left(t_{i}\right)
$$

A function $f: \Omega \rightarrow \mathbb{R}$, where $\Omega$ is an open set in $\mathbb{R}^{p}$, has a directional derivative at $x_{0}$ in $\Omega$, along the direction $v \in \mathbb{R}^{p}$, if the limit

$$
\lim _{h \rightarrow 0^{+}} \frac{f\left(x_{0}+h v\right)-f\left(x_{0}\right)}{h} \equiv d f\left(x_{0} ; v\right)
$$

exists.

If $\mathcal{C}$ is a convex subset of $\Omega$ and if the function $f$ is convex, then for all $x_{0} \in \mathcal{C}$ and for all directions $v \in \mathbb{R}^{p}$, the directional derivative $d f\left(x_{0} ; v\right)$ exists. Furthermore, the following inequalities are valid:

$$
\forall x_{0} \in \mathcal{C}: d f\left(x_{0} ; v\right)+d f\left(x_{0} ;-v\right) \geq 0,
$$

and

$$
\forall\left(x, x_{0}\right) \in \mathcal{C} \times \mathcal{C}: f(x) \geq f\left(x_{0}\right)+d f\left(x_{0} ; x-x_{0}\right) .
$$

Introducing the notion of a scalar product $\langle\cdot \mid \cdot\rangle: \mathbb{R}^{p} \times \mathbb{R}^{p} \rightarrow \mathbb{R}$,

$$
\forall(x, y) \in \mathbb{R}^{p} \times \mathbb{R}^{p}:\langle x \mid y\rangle=\sum_{r=1}^{p} x_{r} y_{r},
$$

and of a metric $\|\cdot\|: \mathbb{R}^{p} \rightarrow \mathbb{R}$,

$$
\forall x \in \mathbb{R}^{p}:\|x\|=\sqrt{\langle x \mid x\rangle},
$$

we can now extend theorems 1 and 2 to higher dimensions.

Theorem 3. Let $f$ be a convex function over a convex set $\mathcal{C}$ in $\mathbb{R}^{p}$. Assuming that $f \in \mathscr{C}^{1}(\mathcal{C})$, we define the function $\tilde{f}$ as

$$
\forall t \in \mathcal{C}: \tilde{f}(t)=\frac{1}{2}\left(\max _{i}\left(f\left(t_{i}\right)+\left\langle\nabla f\left(t_{i}\right) \mid t-t_{i}\right\rangle\right)\right)+\frac{1}{2} \sum_{i=1}^{k} \alpha_{i} f\left(t_{i}\right),
$$

where $t=\sum_{i=1}^{k} \alpha_{i} t_{i}, 0 \leq \alpha_{i} \leq 1$ and $\sum_{i=1}^{k} \alpha_{i}=1$.

Then the function $\tilde{f}$ is an estimate of $f$, which satisfies

$$
|f(c)-\tilde{f}(c)| \leq \frac{1}{2} \max _{i}\left(\alpha_{i}\right) \max _{i, j}\left(\left\|t_{i}-t_{j}\right\|\left\|\nabla f\left(t_{i}\right)-\nabla f\left(t_{j}\right)\right\|\right) .
$$


Theorem 4. Let $f$ be a convex function over a convex set $\mathcal{C}$ in $\mathbb{R}^{p}$. We define the function $\tilde{f}$ as

$$
\forall t \in \mathcal{C}: \tilde{f}(t)=\frac{1}{2}\left(\max _{i}\left(f\left(t_{i}\right)+\left\langle s_{i} \mid t-t_{i}\right\rangle\right)\right)+\frac{1}{2} \sum_{i=1}^{k} \alpha_{i} f\left(t_{i}\right),
$$

where $t=\sum_{i=1}^{k} \alpha_{i} t_{i}, 0 \leq \alpha_{i} \leq 1$ and $\sum_{i=1}^{k} \alpha_{i}=1$ and where $s_{i}$ is any subgradient of $f$ at $t_{i}: \forall i, s_{i} \in \partial f\left(t_{i}\right)$.

Then the function $\tilde{f}$ is an estimate of $f$, which satisfies

$$
|f(c)-\tilde{f}(c)| \leq \frac{1}{2} \max _{i}\left(\alpha_{i}\right) \max _{i, j}\left(\left\|t_{i}-t_{j}\right\|\left\|s_{i}-s_{j}\right\|\right) .
$$

\subsection{Eigenvalues of Hermitian matrices}

The preceding results about convex functions are important in the theory of secular equations for Hermitian matrices due to the following theorem [6, 7].

Theorem 5. Let $\mathcal{H}_{n}$ denote the set of Hermitian $n \times n$ matrices and $\lambda_{1}(H) \geq \cdots \geq$ $\lambda_{n}(H)$ and $X_{k}(H)$ the ordered eigenvalues and the associated normalized eigenvectors, respectively, for any matrix $H$ in $\mathcal{H}_{n}$. The functions $S_{i}: \mathcal{H}_{n} \rightarrow \mathbb{R}$ with $1 \leq i \leq n$, defined by $S_{i}(H)=\sum_{k=1}^{i} \lambda_{k}(H)$, are convex.

The convexity property of the $S_{i}(H)$ implies the concavity property of $\bar{S}_{i}(H)=$ $\sum_{k=i}^{n} \lambda_{k}(H)$ since $\bar{S}_{i}(H)+S_{i-1}(H)=\sum_{k=1}^{n} \lambda_{k}(H)=\operatorname{Tr}(H)$ and the trace satisfies $\operatorname{Tr}\left(\sum_{j} \alpha_{j} H_{j}\right)=\sum_{j} \alpha_{j} \operatorname{Tr}\left(H_{j}\right)$.

\subsection{Spectral projectors of diagonalizable matrices}

If the eigenvalues of a Hermitian matrix are known, the associated eigenvectors can be obtained from the spectral projectors. Two forms of the spectral projector are useful in the following.

Theorem 6. Let $H$ denote a complex $n \times n$ matrix. If $H$ is diagonalizable and if $\lambda_{i}$ with $i=1, \ldots, N$ are its distinct eigenvalues, the spectral projector $\hat{P}_{i}$ associated with $\lambda_{i}$ is given by

$$
\hat{P}_{i}=\frac{1}{\prod_{j \neq i}\left(\lambda_{i}-\lambda_{j}\right)} \sum_{k=0}^{N-1}(-1)^{k}\left(\sum_{\substack{i_{1}<\ldots<i \\ i_{l} \neq i}} \prod_{l=1}^{k} \lambda_{i_{l}}\right) H^{N-1-k} .
$$

Proof. Since $\forall k, 0 \leq k \leq N-1, H^{k}=\sum_{i} \lambda_{i}^{k} \hat{P}_{i}$, the expression for the spectral projectors as a function of powers of $H$ can be deduced by inverting the transpose of a Vandermonde matrix. This inversion can be performed by means of a lower-upper decomposition [9] which gives the expression (14).

Another expression for the spectral projector is given by the following theorem. 
Theorem 7. Let $H$ denote a complex $n \times n$ matrix. If $H$ is diagonalizable and if $\lambda_{i}$ with $i=1, \ldots, N$ are its distinct eigenvalues, the spectral projector $\hat{P}_{i}$ associated with $\lambda_{i}$ is given by

$$
\hat{P}_{i}=\frac{1}{\prod_{j \neq i}\left(\lambda_{i}-\lambda_{j}\right)} \prod_{\substack{j=1 \\ j \neq i}}^{N}\left(H-\lambda_{j} \mathbb{I}\right) .
$$

Theorems 6 and 7 show that, for parameter-dependent matrices, the "singular part" of the spectral operators originates from level crossings and that one may expect abrupt shifts in the eigenvectors when such crossings occur. The interest of the expressions (14) and (15) is that they can be used with approximate expressions $\tilde{\lambda}_{i}$ of eigenvalues as derived from the convexity property stated in theorem 5, to obtain estimates of the spectral projectors.

\section{Convexity in quantum mechanics}

We assume in the following that the Hamiltonian $\tilde{H}$ of an hitherto arbitrary model can be written as a linear combination of $p$ operators $\hat{O}_{j}, \tilde{H}=\sum_{j=1}^{p} c_{j} \hat{O}_{j}$. With the notation $S=\sum_{j}\left|c_{j}\right|$ and $\varepsilon_{j}$ the sign of $c_{j}$, the hamiltonian matrix $\tilde{H}$ can, up to the scaling factor $S$, be rewritten as

$$
\hat{H} \equiv \frac{\tilde{H}}{S}=\sum_{j=1}^{p} \varepsilon_{j} \alpha_{j} \hat{O}_{j}
$$

Since $\alpha_{j}=\left|c_{j}\right| / S$, we have $\alpha_{j} \in[0,1]$ and $\sum_{j} \alpha_{j}=1$. Any sum of the $\hat{O}_{j}$ operators can be rewritten in terms of one of the $2^{p}$ sets of operators $\left\{\hat{H}_{1}^{ \pm}, \ldots, \hat{H}_{p}^{ \pm}\right\}$, with $\hat{H}_{j}^{ \pm} \equiv \pm \hat{O}_{j}$,

$$
\hat{H}=\sum_{j=1}^{p} \alpha_{j} \hat{H}_{j}^{ \pm}=H_{p}^{ \pm}+\sum_{j=1}^{p-1} \alpha_{j}\left(H_{j}^{ \pm}-H_{p}^{ \pm}\right),
$$

which can be seen as the barycentre of the $\hat{H}_{j}^{ \pm}$operators since $\alpha_{j} \in[0,1]$ and $\sum_{j} \alpha_{j}=1$. Therefore, the spectral properties of any Hamiltonian model can be derived from those of sums of $p$ Hermitian $n \times n$ matrices, $H(\vec{\alpha}) \equiv \sum_{j} \alpha_{j} H_{j}$, with $\alpha_{j} \in[0,1]$ and $\sum_{j} \alpha_{j}=1$. The ordered eigenvalues and the associated normalized eigenvectors of $H(\vec{\alpha})$ will be denoted by $\lambda_{1}(\vec{\alpha}) \geq \cdots \geq \lambda_{n}(\vec{\alpha})$ and $X_{k}(\vec{\alpha})$, respectively.

\subsection{Inequalities for the largest or the smallest eigenvalue}

From theorem 5 one deduces that the function $\lambda_{1}(H)$ is convex while $\lambda_{n}(H)$ is concave. Therefore, for all $H_{j} \in \mathcal{H}_{n}$ and for all $\alpha_{j} \in[0,1]$ with $\sum_{j} \alpha_{j}=1$, we have

$$
\begin{aligned}
& \lambda_{1}(\vec{\alpha}) \equiv \lambda_{1}\left(\sum_{j=1}^{p} \alpha_{j} H_{j}\right) \leq \sum_{j=1}^{p} \alpha_{j} \lambda_{1}\left(H_{j}\right), \\
& \lambda_{n}(\vec{\alpha}) \equiv \lambda_{n}\left(\sum_{j=1}^{p} \alpha_{j} H_{j}\right) \geq \sum_{j=1}^{p} \alpha_{j} \lambda_{n}\left(H_{j}\right),
\end{aligned}
$$


corresponding to an upper (lower) limit for the largest (smallest) eigenvalue of $\sum_{j} \alpha_{j} H_{j}$.

From the same convexity (concavity) property, we can also derive a lower (upper) limit for the largest (smallest) eigenvalue of a sum of $p$ Hermitian matrices. To illustrate this derivation, we consider, for simplicity's sake, two matrices, $H_{1}$ and $H_{2}$, both belonging to $\mathcal{H}_{n}$, and their linear combination

$$
H(\alpha)=\alpha H_{1}+(1-\alpha) H_{2},
$$

with $\alpha \in[0,1]$. Furthermore, for any $\left.\alpha^{(0)} \in\right] 0,1\left[\right.$, we introduce $\mathrm{K}_{i} \equiv \operatorname{Ker}\left(H\left(\alpha^{(0)}\right)-\right.$ $\left.\lambda_{i}^{(0)} \mathbb{I}\right)$, the eigenspace of dimension $d_{i}$ associated with the eigenvalue $\lambda_{i}^{(0)} \equiv \lambda_{i}\left(\alpha^{(0)}\right)$.

If $d_{i}=1, \lambda_{i}^{(0)}$ is non-degenerate and it can be shown that its derivative at $\alpha^{(0)}$ exists and is given by

$$
\frac{d \lambda_{i}}{d \alpha}\left(\alpha^{(0)}\right)=X_{i}^{(0) \dagger}\left(H_{1}-H_{2}\right) X_{i}^{(0)}
$$

where $X_{i}^{(0)} \equiv X_{i}\left(\alpha^{(0)}\right)$ is the normalized eigenvector associated with $\lambda_{i}^{(0)}$.

If $d_{i}>1, \lambda_{i}^{(0)}$ is degenerate and the linear variations of the eigenvalue are obtained from stationary perturbation theory by diagonalizing the matrix $H_{1}-H_{2}$ expressed in any orthonormal basis of $\mathrm{K}_{i}$. Let us denote its eigenvalues by $e_{i}^{(1)} \geq \cdots \geq e_{i}^{\left(d_{i}\right)}$. If the largest eigenvalue $\lambda_{1}^{(0)}$ is degenerate, we have that

$$
\begin{aligned}
& \lim _{h \rightarrow 0^{-}} \frac{\lambda_{1}\left(\alpha^{(0)}+h\right)-\lambda_{1}\left(\alpha^{(0)}\right)}{h}=e_{1}^{\left(d_{1}\right)}, \\
& \lim _{h \rightarrow 0^{+}} \frac{\lambda_{1}\left(\alpha^{(0)}+h\right)-\lambda_{1}\left(\alpha^{(0)}\right)}{h}=e_{1}^{(1)},
\end{aligned}
$$

meaning that $e_{1}^{\left(d_{1}\right)}$ and $e_{1}^{(1)}$ are the left and right derivatives of the largest eigenvalue of $H(\alpha)$ at $\alpha^{(0)}$, respectively. Moreover, for any normalized vector $X_{1}^{(0)}$ in $\mathrm{K}_{1}$, we have the property $e_{1}^{\left(d_{1}\right)} \leq X_{1}^{(0) \dagger}\left(H_{1}-H_{2}\right) X_{1}^{(0)} \leq e_{1}^{(1)}$. Therefore, the diagonal matrix element $X_{1}^{(0) \dagger}\left(H_{1}-H_{2}\right) X_{1}^{(0)}$ is larger than the left derivative of the largest eigenvalue and smaller than its right derivative, and the application of the inequality (3) leads to

$$
\lambda_{1}(\alpha) \geq \lambda_{1}^{(0)}+\left(\alpha-\alpha^{(0)}\right) X_{1}^{(0) \dagger}\left(H_{1}-H_{2}\right) X_{1}^{(0)},
$$

for $\left.\alpha^{(0)} \in\right] 0,1[$ and $\alpha \in[0,1]$. This inequality also holds if the largest eigenvalue is non-degenerate since, in this case, the right and the left derivatives are identical, and for $\alpha^{(0)}=0\left(\alpha^{(0)}=1\right)$ where there is only a right (left) derivative.

To summarize, we have found the following range for the largest eigenvalue of $H(\alpha)$ :

$$
\begin{aligned}
& \lambda_{1}(\alpha) \leq \alpha \lambda_{1}\left(H_{1}\right)+(1-\alpha) \lambda_{1}\left(H_{2}\right), \\
& \lambda_{1}(\alpha) \geq \lambda_{1}^{(0)}+\left(\alpha-\alpha^{(0)}\right) X_{1}^{(0) \dagger}\left(H_{1}-H_{2}\right) X_{1}^{(0)},
\end{aligned}
$$

for $\alpha^{(0)}, \alpha \in[0,1]$.

Similarly, for the smallest eigenvalue, due to concavity, the following lower and upper bounds hold:

$$
\begin{aligned}
& \lambda_{n}(\alpha) \geq \alpha \lambda_{n}\left(H_{1}\right)+(1-\alpha) \lambda_{n}\left(H_{2}\right), \\
& \lambda_{n}(\alpha) \leq \lambda_{n}^{(0)}+\left(\alpha-\alpha^{(0)}\right) X_{n}^{(0) \dagger}\left(H_{1}-H_{2}\right) X_{n}^{(0)} .
\end{aligned}
$$


These results can be extended to sums of $p$ Hermitian matrices by use of directional derivatives introduced above. The following lower (upper) bound can then be derived for the largest (smallest) eigenvalue:

$$
\begin{aligned}
& \lambda_{1}(\vec{\alpha}) \geq \lambda_{1}^{(0)}+\sum_{j=1}^{p-1}\left(\alpha_{j}-\alpha_{j}^{(0)}\right) X_{1}^{(0) \dagger}\left(H_{j}-H_{p}\right) X_{1}^{(0)}, \\
& \lambda_{n}(\vec{\alpha}) \leq \lambda_{n}^{(0)}+\sum_{j=1}^{p-1}\left(\alpha_{j}-\alpha_{j}^{(0)}\right) X_{n}^{(0) \dagger}\left(H_{j}-H_{p}\right) X_{n}^{(0)},
\end{aligned}
$$

where $\lambda_{k}^{(0)} \equiv \lambda_{k}\left(\vec{\alpha}^{(0)}\right)$ and $X_{k}^{(0)} \equiv X_{k}\left(\vec{\alpha}^{(0)}\right)$, with $\vec{\alpha}$ representing any $p$ numbers $\alpha_{j} \in[0,1]$ with $\sum_{j} \alpha_{j}=1$.

An important remark is that the eigenvalues of $H(\vec{\alpha})$ are not, in general, differentiable when a level crossing occurs. Indeed, if $e_{1}^{(1)} \neq e_{1}^{\left(d_{1}\right)}$ the eigenvalue $\lambda_{1}(\vec{\alpha})$, degenerate for $\vec{\alpha}=\vec{\alpha}^{(0)}$, has different left and right (in general, directional) derivatives, $\lambda_{1}(\vec{\alpha})$ is not differentiable at $\vec{\alpha}^{(0)}$ and theorem 1 (or its generalized version, theorem 3 ) therefore cannot be applied. However, in this case the quantities $X_{1}^{(0) \dagger}\left(H_{j}-H_{p}\right) X_{1}^{(0)}$ $(j=1, \ldots, p-1)$ interpolate between the directional derivatives such that theorem 2 or theorem 4 can be used to obtain an estimate of the largest eigenvalue.

The inequalities derived in this subsection imply that the range of the largest and smallest eigenvalue of a sum of Hermitian matrices is strongly constrained by the corresponding eigenvalues and eigenvectors of the separate matrices in the sum.

\subsection{Inequalities for the sum of the largest eigenvalues}

The results of the previous section can be generalized to a sum of the largest eigenvalues of Hermitian matrices, which is a convex function. The canonical basis of $\mathbb{R}^{p-1}$ will be denoted by $\vec{e}_{j}(j=1, \ldots, p-1)$.

Theorem 8. Let $H_{j}$ be $p$ Hermitian $n \times n$ matrices and let $H(\vec{\beta})=\sum_{j=1}^{p} \beta_{j} H_{j}$ for all $\vec{\beta} \in \mathbb{R}^{p}$ with $\sum_{j=1}^{p} \beta_{j}=1$ and $\beta_{j} \in[0,1]$. If $\vec{\alpha} \in \mathbb{R}^{p-1}$ denotes the $p-1$ first components of $\vec{\beta}$, then $H(\vec{\beta}) \equiv H(\vec{\alpha})=H_{p}+\sum_{j=1}^{p-1} \alpha_{j}\left(H_{j}-H_{p}\right)$. It is assumed that, for a given $\vec{\alpha}^{(0)} \in \mathbb{R}^{p-1}, H\left(\vec{\alpha}^{(0)}\right)$ has $D$ distinct eigenvalues with multiplicity $d_{l}$. Define $i_{l}$ and $d_{l}$ for $l=1, \ldots, D$ by

$$
\begin{aligned}
& d_{l}=\operatorname{dim}\left(\operatorname{Ker}\left(H\left(\vec{\alpha}^{(0)}\right)-\bar{\lambda}_{l} \mathbb{I}\right)\right), \\
& \bar{\lambda}_{l} \equiv \lambda_{i_{l}}\left(\vec{\alpha}^{(0)}\right)=\lambda_{i_{l}+1}\left(\vec{\alpha}^{(0)}\right)=\cdots=\lambda_{i_{l}+d_{l}-1}\left(\vec{\alpha}^{(0)}\right)>\bar{\lambda}_{l+1} .
\end{aligned}
$$

Introducing $\vec{h}_{j} \equiv h \vec{e}_{j} \in \mathbb{R}^{p-1}$ with $h \in \mathbb{R}$ and $j=1, \ldots, p-1$, we have the properties

$$
\begin{aligned}
& \forall k, 1 \leq k \leq n: \lim _{h \rightarrow 0^{-}} \frac{\lambda_{k}\left(\vec{\alpha}^{(0)}+\overrightarrow{h_{j}}\right)-\lambda_{k}\left(\vec{\alpha}^{(0)}\right)}{h}={ }_{j} e_{l}^{\left(d_{l}-k^{\prime}\right)}\left(\vec{\alpha}^{(0)}\right), \\
& \forall k, 1 \leq k \leq n: \lim _{h \rightarrow 0^{+}} \frac{\lambda_{k}\left(\vec{\alpha}^{(0)}+\overrightarrow{h_{j}}\right)-\lambda_{k}\left(\vec{\alpha}^{(0)}\right)}{h}={ }_{j} e_{l}^{\left(k^{\prime}+1\right)}\left(\vec{\alpha}^{(0)}\right),
\end{aligned}
$$

where $0 \leq k^{\prime} \leq d_{l}-1$ such that $k=i_{l}+k^{\prime}$, and where the ${ }_{j} e_{l}^{(t)}\left(\vec{\alpha}^{(0)}\right)\left(t=1, \ldots, d_{l}\right)$ 
denote the ordered eigenvalues of $\left(H_{j}-H_{p}\right)\left({ }_{j} e_{l}^{(1)} \geq \cdots \geq{ }_{j} e_{l}^{\left(d_{l}\right)}\right)$ within the subspace $\left.\operatorname{Ker}\left(H\left(\vec{\alpha}^{(0)}\right)-\bar{\lambda}_{l} \mathbb{I}\right)\right)$.

Then the two vectors of $\mathbb{R}^{p-1}$ with the following $p-1$ components:

$$
\sum_{\substack{1 \leq k \leq i \\ k=i_{l}+k^{\prime}}} \sum_{\substack{1 \leq l \leq D \\ 0 \leq k^{\prime} \leq d_{l}-1}} e_{l}^{\left(k^{\prime}+1\right)}\left(\vec{\alpha}^{(0)}\right), \quad j=1, \ldots, p-1,
$$

and

$$
\sum_{\substack{1 \leq k \leq i \\ k=i_{l}+k^{\prime}}} \sum_{\substack{1 \leq l \leq D \\ 0 \leq k^{\prime} \leq d_{l}-1}} j e_{l}^{\left(d_{l}-k^{\prime}\right)}\left(\vec{\alpha}^{(0)}\right), \quad j=1, \ldots, p-1,
$$

respectively, are subgradients of $S_{i}(\vec{\alpha}) \equiv \sum_{k=1}^{i} \lambda_{k}(\vec{\alpha})$ at $\vec{\alpha}=\vec{\alpha}^{(0)}$, meaning that

$$
\begin{aligned}
& S_{i}(\vec{\alpha}) \geq S_{i}\left(\vec{\alpha}^{(0)}\right)+\sum_{j=1}^{p-1}\left(\alpha_{j}-\alpha_{j}^{(0)}\right) \sum_{\substack{1 \leq k \leq i \\
k=i_{l}+k^{\prime}}} \sum_{\substack{1 \leq l \leq D \\
0 \leq k^{\prime} \leq d_{l}-1}} j e_{l}^{\left(k^{\prime}+1\right)}\left(\vec{\alpha}^{(0)}\right), \\
& S_{i}(\vec{\alpha}) \geq S_{i}\left(\vec{\alpha}^{(0)}\right)+\sum_{j=1}^{p-1}\left(\alpha_{j}-\alpha_{j}^{(0)}\right) \sum_{\substack{1 \leq k \leq i \\
k=i_{l}+k^{\prime}}} \sum_{\substack{1 \leq l \leq D \\
0 \leq k^{\prime} \leq d_{l}-1}} e_{l}^{\left(d_{l}-k^{\prime}\right)}\left(\vec{\alpha}^{(0)}\right),
\end{aligned}
$$

for all $\vec{\alpha} \in[0,1]^{p-1}$ with $\sum_{j=1}^{p-1} \alpha_{j} \leq 1$.

It has to be noted that Ye and Hiriart-Urruty [10] have obtained a similar result while deriving the general expression of the subdifferential of a symmetric matrix.

\subsection{Estimates of eigenvalues}

Using the same notations as in the previous theorem, let us assume that the Hamiltonian $H(\vec{\alpha})$ has been diagonalized for $P \geq p$ vectors $\vec{\alpha}^{(v)}(v=1, \ldots, P), p-1$ among them being independent. We introduce the following functions $m_{i}(\vec{\alpha})$ and $M_{i}(\vec{\alpha})$ :

$$
m_{i}(\vec{\alpha}) \equiv \max _{v=1, \ldots, P}\left(S_{i}^{(v)}+\sum_{j=1}^{p-1} p_{i j}^{(v)}\left(\alpha_{j}-\alpha_{j}^{(v)}\right), S_{i}^{(v)}+\sum_{j=1}^{p-1} q_{i j}^{(v)}\left(\alpha_{j}-\alpha_{j}^{(v)}\right)\right),
$$

with $S_{i}^{(v)} \equiv S_{i}\left(\vec{\alpha}^{(v)}\right)$ and where

$$
\begin{aligned}
& p_{i j}^{(v)}=\sum_{\substack{1 \leq k \leq i \\
1 \leq i_{l}^{(v)}+k^{\prime}}} \sum_{\substack{1 \leq l \leq D^{(v)} \\
0 \leq k^{\prime} \leq d_{l}^{(v)}-1}} e_{l}^{\left(k^{\prime}+1\right)}\left(\vec{\alpha}^{(v)}\right), \\
& q_{i j}^{(v)}=\sum_{\substack{1 \leq k \leq i \\
k=i_{l}^{(v)}+k^{\prime}}} \sum_{\substack{1 \leq l \leq D^{(v)} \\
0 \leq k^{\prime} \leq d_{l}^{(v)}-1}} j e_{l}^{\left(d_{l}^{(v)}-k^{\prime}\right)}\left(\vec{\alpha}^{(v)}\right),
\end{aligned}
$$

and

$$
M_{i}(\vec{\alpha}) \equiv \min _{\left\{v_{j}\right\} \in I} \sum_{j=1}^{p} \gamma_{j} S_{i}^{\left(v_{j}\right)}
$$


where $I$ is the set of labels for which $\vec{\alpha}$ can be written as a barycentre of some $\vec{\alpha}^{\left(v_{j}\right)}$ $(j=1, \ldots, p)$, and where the $\gamma_{j}$ are the barycentric coordinates of $\vec{\alpha}$ within this new frame, as explained below. If the functions $\tilde{S}_{i}$ are defined as

$$
\tilde{S}_{i}(\vec{\alpha}) \equiv \frac{1}{2}\left(M_{i}(\vec{\alpha})+m_{i}(\vec{\alpha})\right)
$$

theorem 4 applies to $\left|S_{i}(\vec{\alpha})-\tilde{S}_{i}(\vec{\alpha})\right|$. We have therefore obtained the following estimates $\tilde{\lambda}_{i}$ of the eigenvalues $\lambda_{i} \equiv S_{i}-S_{i-1}$ :

$$
\tilde{\lambda}_{i}(\vec{\alpha}) \equiv \frac{1}{2}\left(M_{i}(\vec{\alpha})+m_{i}(\vec{\alpha})-M_{i-1}(\vec{\alpha})-m_{i-1}(\vec{\alpha})\right) .
$$

A geometrical way of computing $M_{i}(\vec{\alpha})$ is illustrated in figure 2 , for $p=3$ and $P=10$. It is assumed that the convex function $S_{i}(\vec{\alpha})$ is known for $P$ choices of $\vec{\alpha}$ : these points $\vec{\alpha}^{(v)}(v=1, \ldots, P)$, which are interpreted as barycentric coordinates of points within an equilateral triangle, are shown as the blue crosses in figure 2 . The associated points $\left(\vec{\alpha}^{(v)}, S_{i}^{(v)}\right)$, which belong to $\mathbb{R}^{p}$, are represented by the blue squares. The aim is to compute the value of $M_{i}(\vec{\alpha})$ for a given $\vec{\alpha}$ represented by the red cross, and this is done in the following way. From the blue crosses several triangles can be built by choosing $p$ points among the $\vec{\alpha}^{(v)}$. Each triangle is characterized by a set $\vec{\alpha}^{\left(v_{j}\right)}(j=1, \ldots, p)$, and some of these triangles contain the red cross. The set of all triangles containing $\vec{\alpha}$ defines the set $I$ in equation (25). Two examples of such triangles are represented in figure 2 by the black and green dotted triangles. If $\left\{v_{j}\right\} \in I, \vec{\alpha}$ is inside the associated triangle and can be written as a barycentre of the vertices with the barycentric coordinates $\gamma_{j}$ appearing in equation (25). Due to convexity one therefore has that $S_{i}(\vec{\alpha}) \leq \sum_{j=1}^{p} \gamma_{j} S_{i}^{\left(v_{j}\right)}$. The triangles in black and green full lines in figure 2 illustrate the computation of $\sum_{j=1}^{p} \gamma_{j} S_{i}^{\left(v_{j}\right)}$ for the two different choices of the triangle. The minimum value of $\sum_{j=1}^{p} \gamma_{j} S_{i}^{\left(v_{j}\right)}$ for all $\left\{v_{j}\right\} \in I$ (which are represented by the purple crosses) yields $M_{i}(\vec{\alpha})$. Finally, the solid red curve shows the $M_{i}(\vec{\alpha})$ obtained in this way for several values of $\vec{\alpha}$.

These estimates of the eigenvalues can now be used to approximate the spectral projector using the expressions given in theorems 6 or 7 . It can be noted that, in practice, the calculation of powers of $H(\vec{\alpha})$ (which might be time consuming) can be deduced from $\vec{\alpha}$, from the powers of eigenvalues of $H_{i}$ and from their spectral projectors, which need to be computed only once.

\section{Application to the vibron model}

In this section we apply the estimates derived in the previous sections to the vibron model of molecules. The vibron model was proposed by Iachello and Levine to study molecular rotation-vibration spectra, in particular of diatomic molecules. Many of the essential features of such molecules can be characterized in terms of the distance vector $\vec{r}$ between the atoms which behaves as a rank-1 tensor under rotations and which has negative parity. It is therefore natural to introduce operators with similar transformation properties, which leads to a $\mathrm{U}(4)$ realization in terms of a vector (rank-1) operator with 


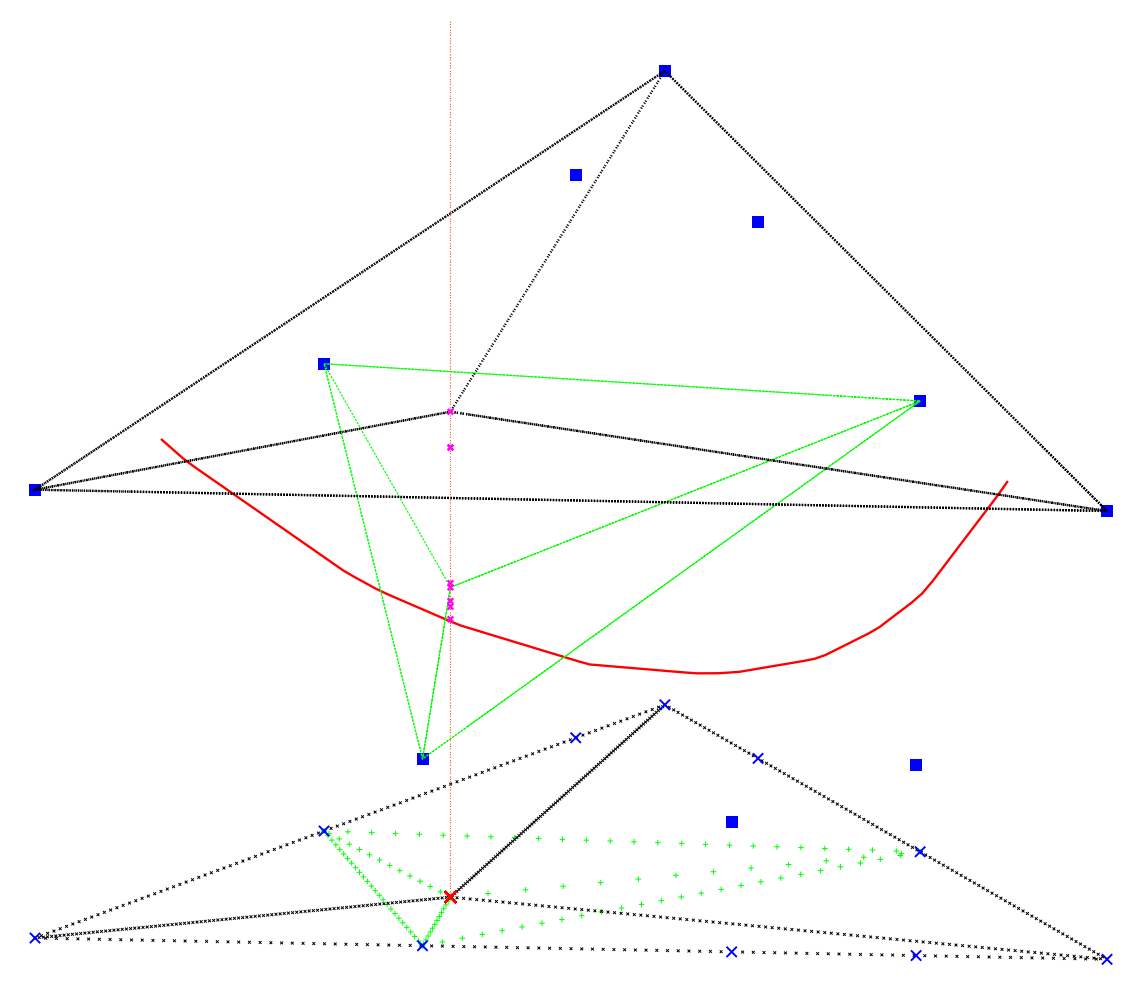

Figure 2. Graphical illustration of the computation of $M_{i}(\vec{\alpha})$ in equation (25). Symbols in the figure are explained in the text.

components $p_{m}^{\dagger}$ and a scalar (rank-0) operator $s^{\dagger}$. Since these operators have integer angular momentum, they create bosonic states. While the $p$ boson is used to describe the dipole degree of freedom, the physical role of the $s$ boson is less clear; its introduction is justified mainly on phenomenological grounds as a way to generate interactions between bosonic states with different numbers of $p$ bosons. A detailed description of the vibron model can be found in [11].

The $p$ and $s$ bosons are the basic building blocks of the $\mathrm{U}(4)$ molecular vibron model, which is taken here as a testing ground of our previously derived eigenvalue estimates.

\subsection{The vibron model with two-body interactions}

A simple one- plus two-body Hamiltonian of the vibron model takes the following form:

$$
\tilde{H}_{\text {vibron }}=\alpha \mathcal{C}_{U(3)}+\beta \mathcal{C}_{S O(4)}+\gamma \mathcal{C}_{S O(3)},
$$

where $\mathcal{C}_{G}$ is a quadratic Casimir operator of the algebra $G \subset U(4)$ with eigenvalues and eigenvectors that can be derived analytically [12]. With $S=|\alpha|+|\beta|+|\gamma|$ and $\alpha_{1}=|\beta| / S, \alpha_{2}=|\gamma| / S$ and $\alpha_{3}=|\alpha| / S$, the scaled vibron Hamiltonian becomes

$$
\begin{aligned}
\hat{H}_{\text {vibron }}^{\varepsilon_{1}, \varepsilon_{2}, \varepsilon_{3}}\left(\alpha_{1}, \alpha_{2}\right) & =\varepsilon_{3} \mathcal{C}_{U(3)}+\alpha_{1}\left(\varepsilon_{1} \mathcal{C}_{S O(4)}-\varepsilon_{3} \mathcal{C}_{U(3)}\right) \\
& +\alpha_{2}\left(\varepsilon_{2} \mathcal{C}_{S O(3)}-\varepsilon_{3} \mathcal{C}_{U(3)}\right)
\end{aligned}
$$



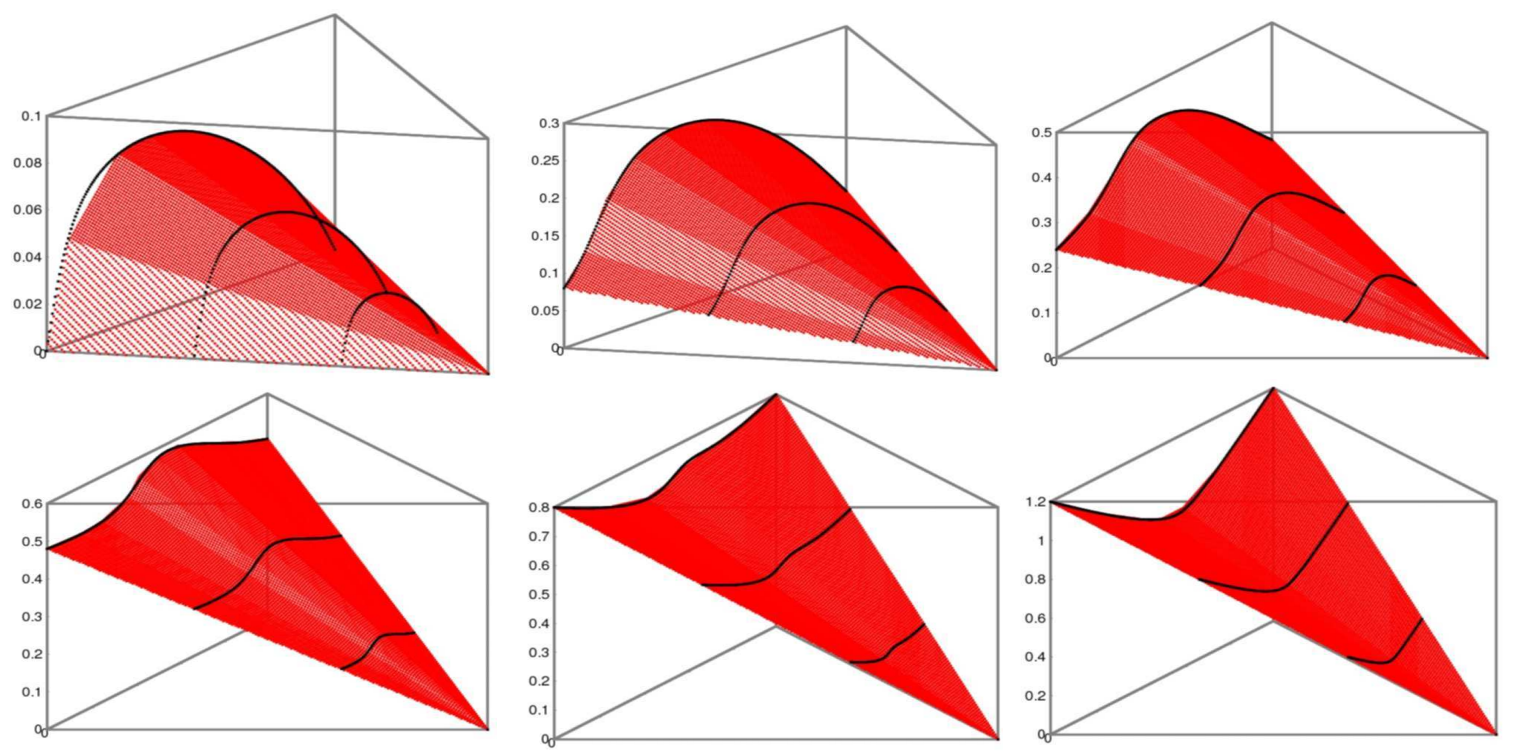

Figure 3. The red surfaces represent the estimates of (a) the lowest, (b) the second, (c) the third, (d) the fourth, (e) the fifth and (f) the largest eigenvalue obtained with the vibron Hamiltonian (28) for $N=10$ bosons, for angular momentum $L=0$ and for $\left(\varepsilon_{1}, \varepsilon_{2}, \varepsilon_{3}\right)=(+,+,+)$. The black curves represent the exact eigenvalues for $\alpha_{2}=0$, $1 / 3$ and $2 / 3$, and $\alpha_{1} \in\left[0,1-\alpha_{2}\right]$.

with $\varepsilon_{i}= \pm, \alpha_{i} \in[0,1]$ and $\alpha_{1}+\alpha_{2} \leq 1$. Since the Hamiltonian is the sum of three operators, $2^{3}$ classes of spectra are to be studied, corresponding to the eight choices of $\left(\varepsilon_{1}, \varepsilon_{2}, \varepsilon_{3}\right)$.

We calculate the eigenvalues and eigenvectors for the following six sets of $\vec{\alpha}^{(0)} \equiv$ $\left(\alpha_{1}^{(0)}, \alpha_{2}^{(0)}\right)$ for a given boson number $N$ :

- for $\vec{\alpha}^{(0)}=(0,0), H\left(\vec{\alpha}^{(0)}\right)=\mathcal{C}_{U(3)}$ corresponds to the $U(3)$ limit and describes a non-rigid molecule,

- for $\vec{\alpha}^{(0)}=(1,0), H\left(\vec{\alpha}^{(0)}\right)=\mathcal{C}_{S O(4)}$ corresponds to the $S O(4)$ limit and describes a rigid molecule,

- for $\vec{\alpha}^{(0)}=(0,1), H\left(\vec{\alpha}^{(0)}\right)=\mathcal{C}_{S O(3)}$,

- and for $\vec{\alpha}^{(0)}=(1 / 4,0),(1 / 2,0)$ and $(3 / 4,0)$, the spectra are obtained by numerical diagonalization.

From the spectra obtained for these six values of $\vec{\alpha}^{(0)}$ and for any angular momentum $L$, we can determine the surfaces defined by the estimates of the eigenvalues for any $\vec{\alpha}$. These surfaces are plotted in figure 3 for angular momentum $L=0$ and $\left(\varepsilon_{1}, \varepsilon_{2}, \varepsilon_{3}\right)=(+,+,+)$. On the three-dimensional plots in figure 3 , each point is defined by $\left(\alpha_{1}, \alpha_{2}, \lambda\right)$, where the $\left(\alpha_{1}, \alpha_{2}\right)$ are interpreted as the barycentric coordinates of a point inside an equilateral triangle and $\lambda$ is the eigenvalue. For comparison, each panel also shows the numerically computed exact eigenvalues for $\alpha_{2}=0,1 / 3$ and $2 / 3$, and for all $\alpha_{1} \in\left[0,1-\alpha_{2}\right]$.

The quality of the approximation of the exact eigenvalues is further illustrated in 

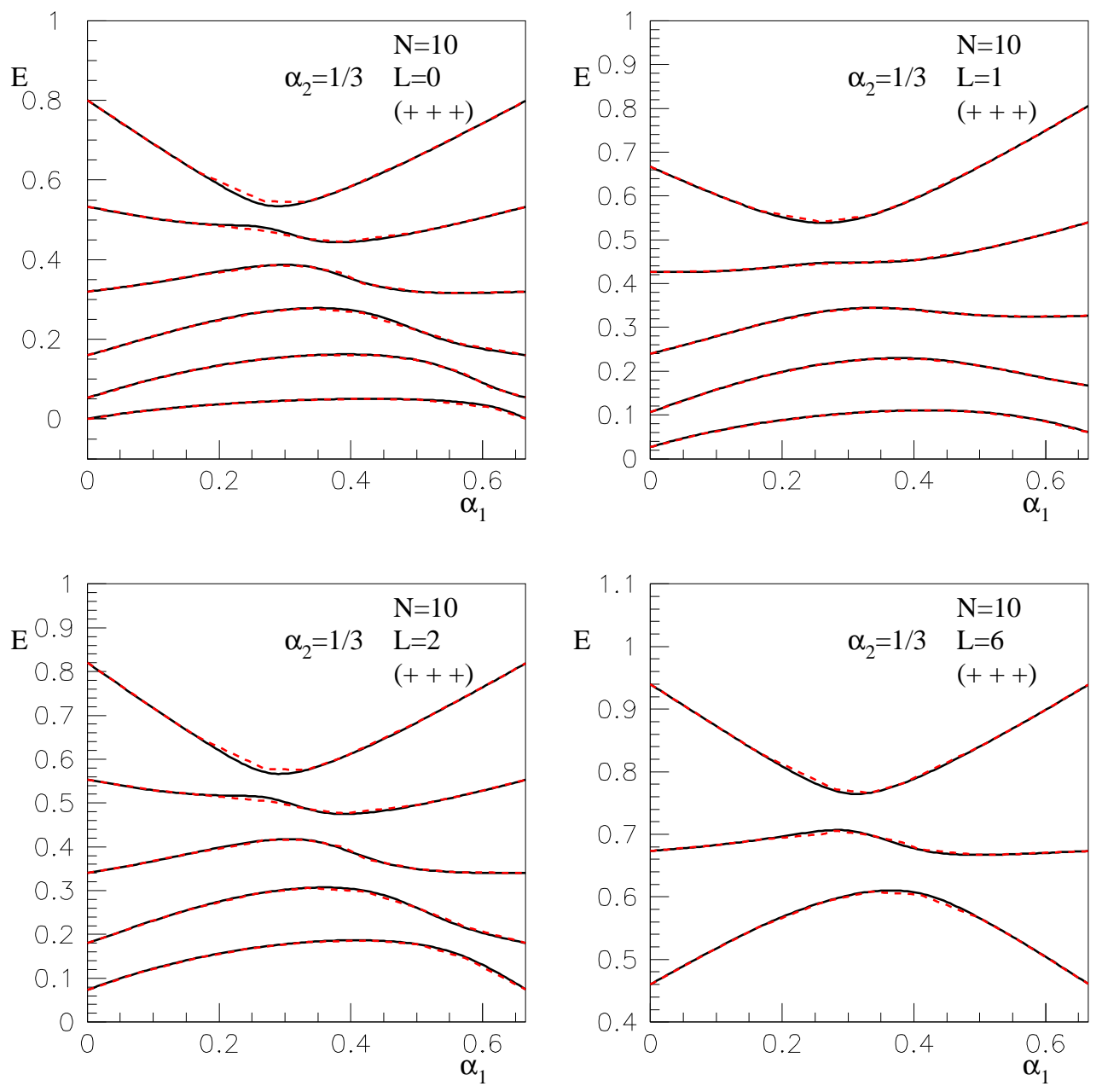

Figure 4. Energy spectra of the vibron Hamiltonian (28) for $N=10$ bosons, for angular momenta $L=0,1,2$ and 6 , and for $\left(\varepsilon_{1}, \varepsilon_{2}, \varepsilon_{3}\right)=(+,+,+)$. The black curves represent the exact eigenvalues for $\alpha_{2}=1 / 3$ and $\alpha_{1} \in[0,2 / 3]$ while the dashed red lines are the estimates of the eigenvalues for each $L$.

figure 4 , which shows vibron spectra for $N=10$ bosons and for various angular momenta $L$, with $\alpha_{2}=1 / 3$ and for all $\alpha_{1} \in[0,2 / 3]$.

\subsection{Ground-state angular momentum}

We now apply the eigenvalue estimates to the problem of the dominance of certain angular-momentum states in the vibron model. In many quantum-mechanical models it was observed that, even if random interactions among the constituent particles are adopted, the ground state has a preference for certain angular momenta. This was first pointed out by Johnson et al. [13, 14] in the context of the nuclear shell model (see 

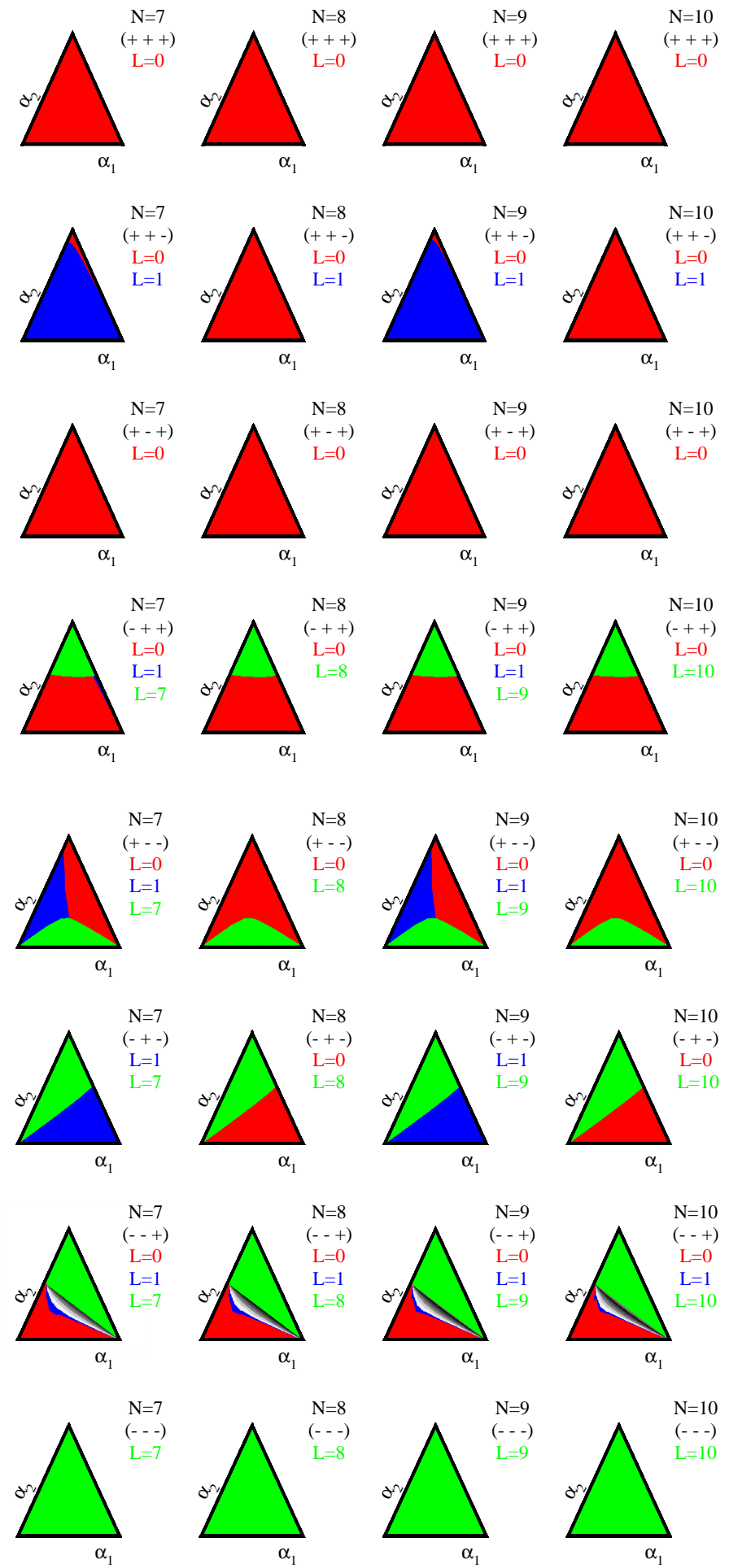

Figure 5. Angular momentum $L$ for $N=7,8,9$ and 10 bosons with the vibron Hamiltonian (28), as derived from the comparison of the estimates of the lowest eigenvalues. If the angular momentum of the estimate of the eight classes of Hamiltonians $\hat{H}_{\text {vibron }}^{\varepsilon_{1}, \varepsilon_{2}, \varepsilon_{3}}\left(\alpha_{1}, \alpha_{2}\right)$ (with $\varepsilon_{i}= \pm$ ) is $L=0,1$ or $N$, the pixel at the point $\left(\alpha_{1}, \alpha_{2}\right)$ in the triangle is red, blue or green, respectively. The grey pixels represent points for which $2 \leq L \leq N-1$. 

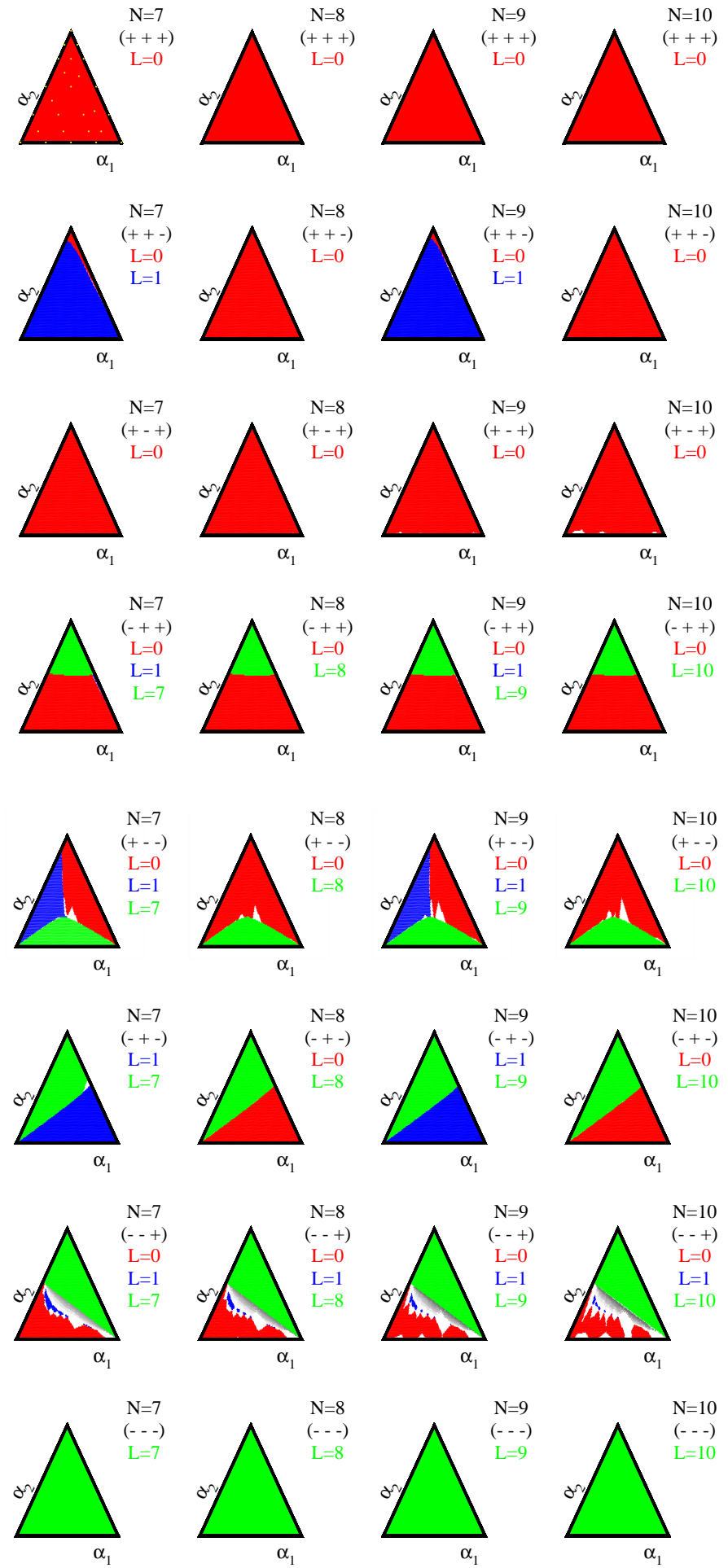

Figure 6. Ground-state angular momentum $L$ for $N=7,8,9$ and 10 bosons with the vibron Hamiltonian (28), as derived from the inequalities (22) and (21) of the lowest eigenvalues. If the angular momentum of the ground state of the eight classes of Hamiltonians $\hat{H}_{\text {vibron }}^{\varepsilon_{1}, \varepsilon_{2}, \varepsilon_{3}}\left(\alpha_{1}, \alpha_{2}\right)$ (with $\varepsilon_{i}= \pm$ ) is $L=0,1$ or $N$, the pixel at the point $\left(\alpha_{1}, \alpha_{2}\right)$ in the triangle is red, blue or green, respectively. In white areas no definite ground-state angular momentum could be deduced from the inequalities. The yellow dots on the first triangle indicate the points used to derive the inequalities. 
also [15]) and subsequently shown to apply to other models of nuclei as well [16, 17]. It was also observed to be valid in the vibron model, where the angular-momentum preponderance was successfully explained with coherent-state theory [18]. The problem has received extensive attention since then (see, e.g. [19]) but arguably no comprehensive explanation of the observed preponderance has come forward. We show here that the eigenvalue estimates derived in the previous section can be used to derive the angularmomentum preponderance in the vibron model from a limited number of spectra.

Figure 5 displays the angular momenta $L$ associated with the lowest $\tilde{\lambda}_{L}$ of the eight classes of Hamiltonians $\hat{H}_{\text {vibron }}^{\varepsilon_{1}, \varepsilon_{2}, \varepsilon_{3}}\left(\alpha_{1}, \alpha_{2}\right)$ with $\varepsilon_{i}= \pm$, for $N=7,8,9$ and 10 bosons with $L=0,1$ or $N$ corresponding to red, blue or green, respectively. The grey dots denote angular momenta ranging from 2 to $N-1$ : the higher $L$, the darker is the dot. The ground-state angular momentum can be determined exactly from the inequalities (22) and (21). Since, in some cases, no angular momentum could be assigned due to the range of these inequalities using six points, we have performed calculations with twentysix points which are represented by the yellows dots on the left triangle of figure 6 . On this figure, we have used the same colours as in figure 5 to display the ground-state angular momenta $L$ of the eight classes of Hamiltonians $\hat{H}_{\text {vibron }}^{\varepsilon_{1}, \varepsilon_{2}, \varepsilon_{3}}\left(\alpha_{1}, \alpha_{2}\right)$ with $\varepsilon_{i}= \pm$, for $N=7,8,9$, and 10 bosons. If no ground-state angular momentum could be derived from the inequalities, the point is left white.

From the property of convexity and from the calculation of only twenty-six different spectra, we can deduce the following ground-state properties:

- A large fraction of the parameter space yields a ground-state with $L=0$ (red), notably for $\left(\varepsilon_{1}, \varepsilon_{2}, \varepsilon_{3}\right)=(+,+,+),(-,+,+)$ and $(+,-,+)$.

- The ground-state angular momentum is always stretched, $L=N$ (green), for $\left(\varepsilon_{1}, \varepsilon_{2}, \varepsilon_{3}\right)=(-,-,-)$, and the component of the same configuration is large for $\left(\varepsilon_{1}, \varepsilon_{2}, \varepsilon_{3}\right)=(+,-,-)$ and $(-,-,+)$.

- For $\left(\varepsilon_{1}, \varepsilon_{2}, \varepsilon_{3}\right)=(+,+,-)$ the ground-state angular momentum oscillates between $L=1$ and $L=0$ depending on whether the number of bosons $N$ is odd or even. This odd-even staggering effect is also partly present in the $\left(\varepsilon_{1}, \varepsilon_{2}, \varepsilon_{3}\right)=(+,-,-)$ and $(-,+,-)$ spectra.

\subsection{The vibron model with a three-body interaction}

To illustrate the versatility of our eigenvalue estimates, we generalize the Hamiltonian of the vibron model and replace the rather trivial angular-momentum term $\mathcal{C}_{S O(3)}$ by a three-body interaction, leading to the Hamiltonian

$$
{ }^{3 \mathrm{~b}} \tilde{H}_{\text {vibron }}=\alpha \mathcal{C}_{U(3)}+\beta \mathcal{C}_{S O(4)}+\gamma \mathcal{C}_{3 \mathrm{~b}}
$$

where $\mathcal{C}_{3 \mathrm{~b}}$ is defined by

$$
\begin{aligned}
\mathcal{C}_{3 \mathrm{~b}}= & {\left[p^{\dagger} \times p^{\dagger} \times p^{\dagger}\right]^{(3)} \cdot[\tilde{p} \times \tilde{p} \times \tilde{p}]^{(3)} } \\
& +\left[\left[p^{\dagger} \times p^{\dagger}\right]^{(0)} \times s^{\dagger}\right]^{(0)} \cdot[\tilde{s} \times \tilde{s} \times \tilde{s}]^{(0)}+\text { h.c. }
\end{aligned}
$$


This operator conserves angular momentum but not the number of $p$ bosons. Similar to the discussion of the two-body vibron Hamiltonian, we define $S=|\alpha|+|\beta|+|\gamma|$ and $\alpha_{1}=|\beta| / S, \alpha_{2}=|\gamma| / S$ and $\alpha_{3}=|\alpha| / S$. The scaled three-body vibron Hamiltonian then reads

$$
\begin{aligned}
{ }^{3 \mathrm{~b}} \hat{H}_{\text {vibron }}^{\varepsilon_{1}, \varepsilon_{2}, \varepsilon_{3}}\left(\alpha_{1}, \alpha_{2}\right) & =\varepsilon_{3} \mathcal{C}_{U(3)}+\alpha_{1}\left(\varepsilon_{1} \mathcal{C}_{S O(4)}-\varepsilon_{3} \mathcal{C}_{U(3)}\right) \\
& +\alpha_{2}\left(\varepsilon_{2} \mathcal{C}_{3 \mathrm{~b}}-\varepsilon_{3} \mathcal{C}_{U(3)}\right),
\end{aligned}
$$

with $\varepsilon_{i}= \pm, \alpha_{i} \in[0,1]$ and $\alpha_{1}+\alpha_{2} \leq 1$. We can now study ground-state properties of this Hamiltonian by using the concavity property of the smallest eigenvalue. The spectra are computed for the following 10 values of $\vec{\alpha}^{(0)}$ :

- for $\vec{\alpha}^{(0)}=(0,0), H\left(\vec{\alpha}^{(0)}\right)=\mathcal{C}_{U(3)}$,

- for $\vec{\alpha}^{(0)}=(1,0), H\left(\vec{\alpha}^{(0)}\right)=\mathcal{C}_{S O(4)}$,

- for $\vec{\alpha}^{(0)}=(0,1), H\left(\vec{\alpha}^{(0)}\right)=\mathcal{C}_{3 \mathrm{~b}}$,

- and for 7 other values of $\vec{\alpha}^{(0)}$ which have been obtained iteratively to minimize the distance beween the lower and upper surfaces for $L=0$ defined by the inequalities (19) and (21). In these 7 points the spectra are obtained by numerical diagonalization.

These 10 spectra are used to obtain estimates for the eigenvalues within the entire parameter space for a given angular momentum and a given boson number.

The quality of the approximation of the eigenvalues is illustrated in figures 7 and 8, which show spectra of the three-body vibron Hamiltonian (29) for $N=10$ bosons, for various angular momenta $L$, with $\alpha_{2}=1 / 3$, for all $\alpha_{1} \in[0,2 / 3]$, and for $\left(\varepsilon_{1}, \varepsilon_{2}, \varepsilon_{3}\right)=(+,+,+)$ and $(+,-,+)$, respectively. The red curves denote the exact eigenvalues while the black ones represent the estimates deduced from the 10 spectra.

We illustrate the accuracy of our method further by comparing exact eigenvectors of the three-body vibron Hamiltonian (29) with those obtained with a spectral projector with approximate expressions for the eigenvalues. In figure 9 are shown, for the two cases $\left(\varepsilon_{1}, \varepsilon_{2}, \varepsilon_{3}\right)=(+,+,+)$ and $(+,-,+)$, the absolute values of the components of the eigenvector $\left|0_{1}\right\rangle$, associated with the lowest eigenvalue of the Hamiltonian (29) for $N=10$ bosons, for angular momentum $L=0$ and for $\alpha_{2}=1 / 3$ and $\alpha_{1} \in[0,2 / 3]$. It is seen that the exact results in black are close to the approximate ones (red), deduced from the spectral projectors with the interpolated eigenvalues. An even more detailed test is carried out in figure 10 where the absolute value of the third component of the approximate eigenvector $\left|0_{1}\right\rangle$ is shown (red surface) for all $\alpha_{1} \in[0,1]$ and $\alpha_{2} \in[0,1]$. For comparison, the exact component for $\alpha_{2}=0,1 / 3$ and $2 / 3$, and for all $\alpha_{1} \in\left[0,1-\alpha_{2}\right]$ is shown in black.

We can now revisit the problem of the ground-state angular momentum with the three-body vibron Hamiltonian (29). Figure 11 displays the angular momenta $L$ of the lowest $\tilde{\lambda}_{L}$ of the eight classes of Hamiltonians ${ }^{3 \mathrm{~b}} \hat{H}_{\mathrm{vibron}}^{\varepsilon_{1}, \varepsilon_{2}, \varepsilon_{3}}\left(\alpha_{1}, \alpha_{2}\right)$ with $\varepsilon_{i}= \pm$, for $N=7,8,9$ and 10 bosons with $L=0,1$ or $N$ corresponding to red, blue or green, respectively. The inequalities (21) and (22) with the ten-point estimates lead to rather 

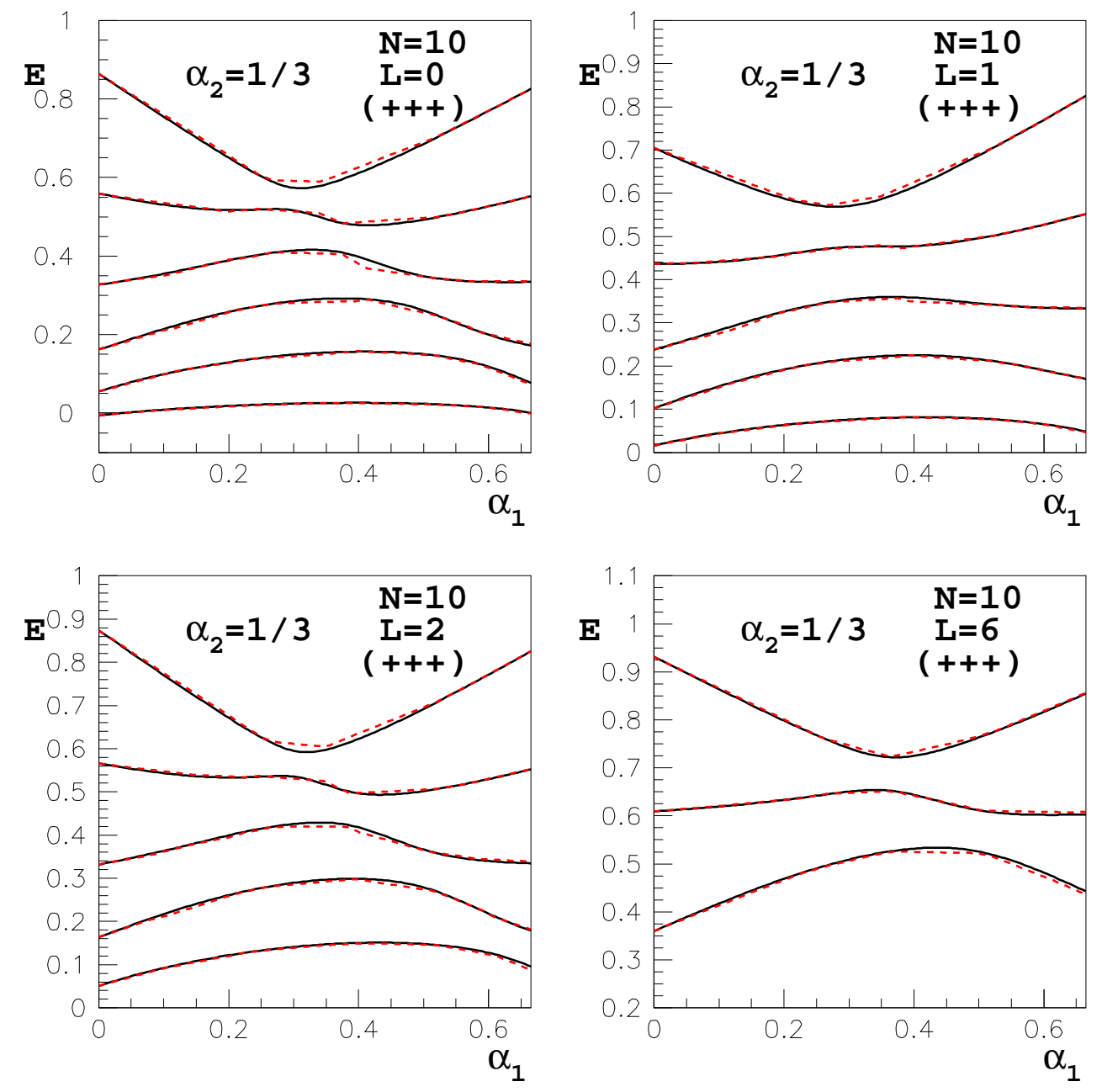

Figure 7. Energy spectra of the three-body vibron Hamiltonian (29) for $N=10$ bosons, for angular momenta $L=0,1,2$ and 6 , and for $\left(\varepsilon_{1}, \varepsilon_{2}, \varepsilon_{3}\right)=(+,+,+)$. The black curves represent the exact eigenvalues for $\alpha_{2}=1 / 3$ and $\alpha_{1} \in[0,2 / 3]$ while the red dashed lines are the interpolation deduced from the calculation of 10 spectra.

large inconclusive regions. Therefore, we have also performed calculations using twentysix points. The results are shown in figure 12 .

We observe the following effects of the three-body interaction on the ground-state properties:

- For $\left(\varepsilon_{1}, \varepsilon_{2}, \varepsilon_{3}\right)=(+,-,+)$ : Due to the three-body interaction, states with $L \geq 1$ compete with those with $L \geq 0$ to become the ground state, a phenomenon which is not observed for two-body interactions.

- For $\left(\varepsilon_{1}, \varepsilon_{2}, \varepsilon_{3}\right)=(+,-,-)$ : While for the two-body vibron Hamiltonian the groundstate angular momentum is $L=0$ for a wide parameter region, this is no longer true 

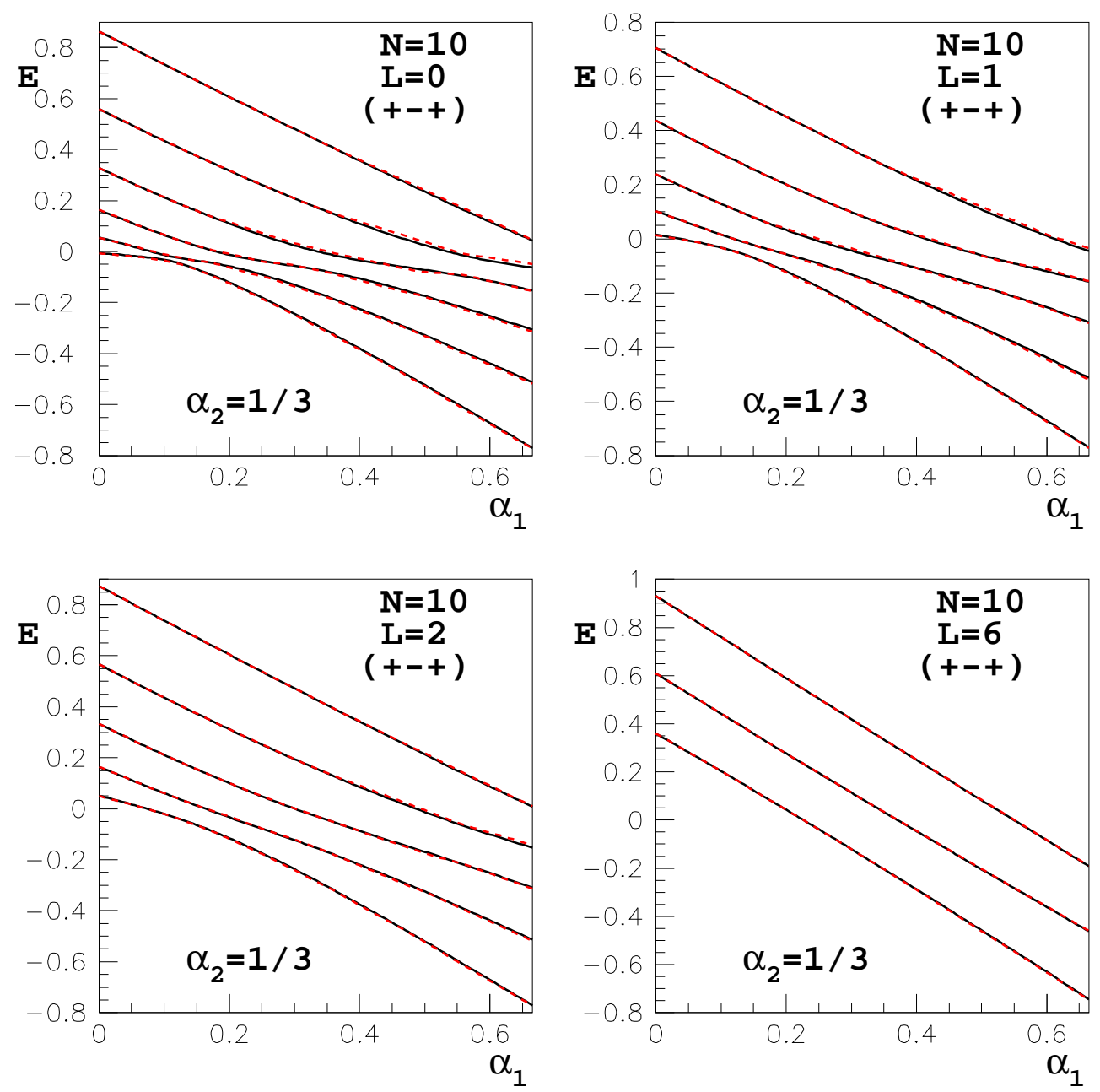

Figure 8. Same caption as figure 7 for $\left(\varepsilon_{1}, \varepsilon_{2}, \varepsilon_{3}\right)=(+,-,+)$.

for the three-body vibron Hamiltonian and this configuration is now competiting with other angular momenta.

- For $\left(\varepsilon_{1}, \varepsilon_{2}, \varepsilon_{3}\right)=(-,+,-)$ : The ground-state region with angular momentum $L=N(L=0$ or $L=1)$ reduces (increases) in size.

Level crossings, which are often a signature of a quantum phase transition [20], can also be predicted with the present technique. Indeed, if such crossing occurs for the levels $i$ and $i+1$, then the ratiof $\left(\lambda_{1}-\lambda_{n}\right) /\left(\lambda_{i}-\lambda_{i+1}\right)(1 \leq i \leq n-1)$ should exhibit a peak and, therefore, the localization of peaks in the approximate ratio $\left(\tilde{\lambda}_{1}-\tilde{\lambda}_{n}\right) /\left(\tilde{\lambda}_{i}-\tilde{\lambda}_{i+1}\right)$ will be a signature of a phase transition. This is exemplified in figures 13 and 14, which show results for the three-body vibron Hamiltonian (29). On the left panel of figure 13 $\ddagger$ The numerator in this expression is introduced to remove effects coming from spectrum dilatation or compression. 

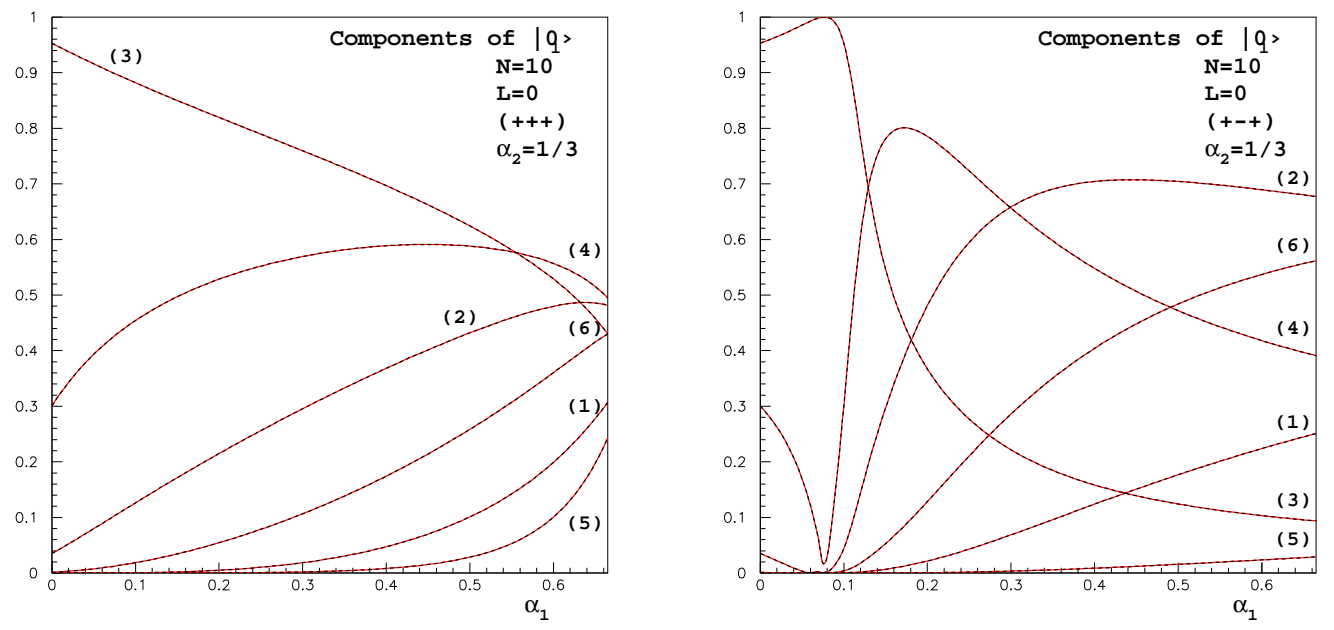

Figure 9. Absolute values of the components of the eigenvector $\left|0_{1}\right\rangle$, associated with the lowest eigenvalue of the vibron Hamiltonian (29) for $N=10$ bosons, for angular momentum $L=0$, and for $\alpha_{2}=1 / 3$ and $\alpha_{1} \in[0,2 / 3]$. The exact (approximate) results are shown in black (red). The left and right panels correspond to $\left(\varepsilon_{1}, \varepsilon_{2}, \varepsilon_{3}\right)=(+,+,+)$ and $(+,-,+)$, respectively.

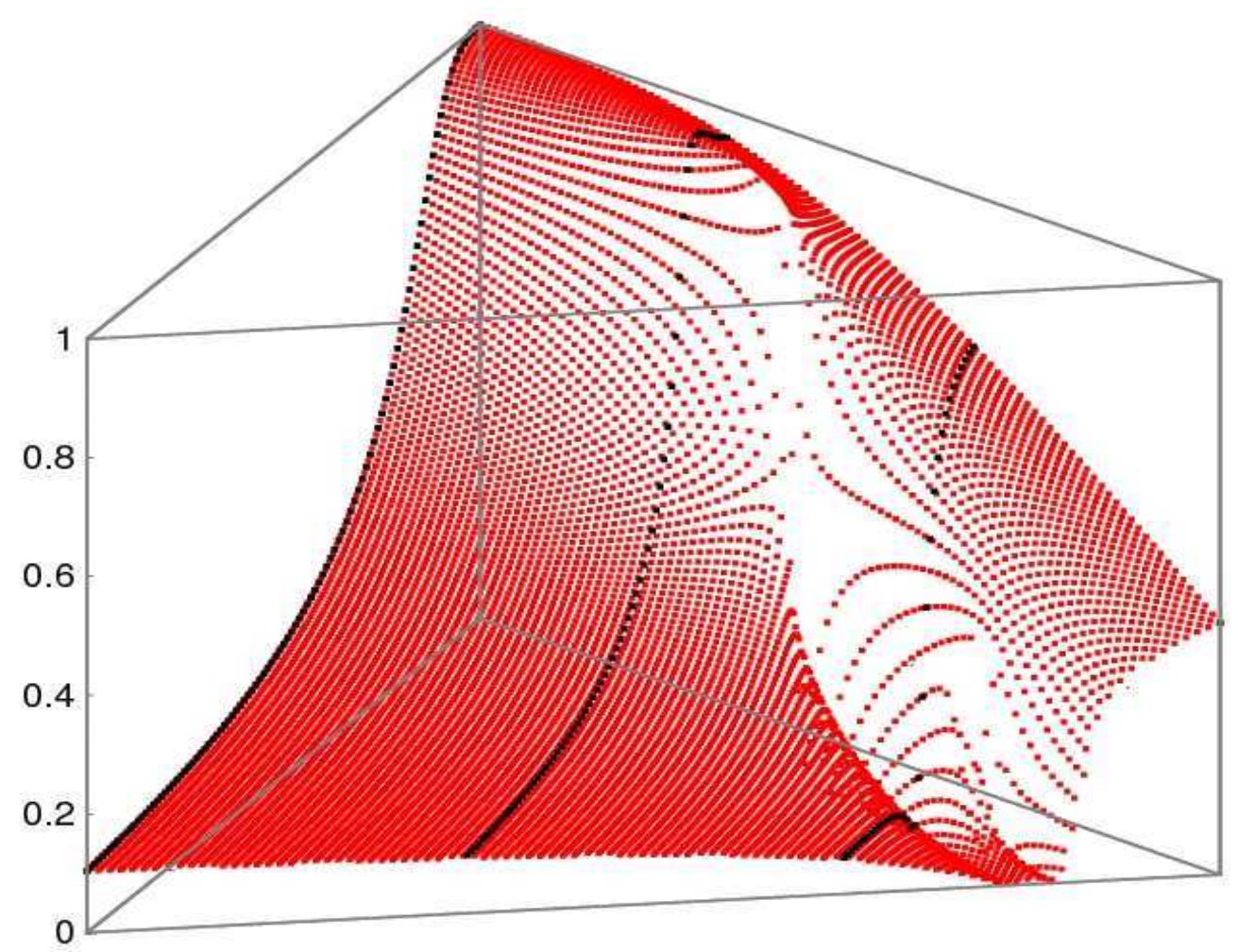

Figure 10. Absolute value of the third component of the eigenvector $\left|0_{1}\right\rangle$, associated with the lowest eigenvalue of the vibron Hamiltonian (29) for $N=10$ bosons and for angular momentum $L=0$. The red surface shows the approximate value for all $\alpha_{1} \in[0,1]$ and $\alpha_{2} \in[0,1]$ while the exact component for $\alpha_{2}=0,1 / 3$ and $2 / 3$, and for all $\alpha_{1} \in\left[0,1-\alpha_{2}\right]$ is shown in black. 

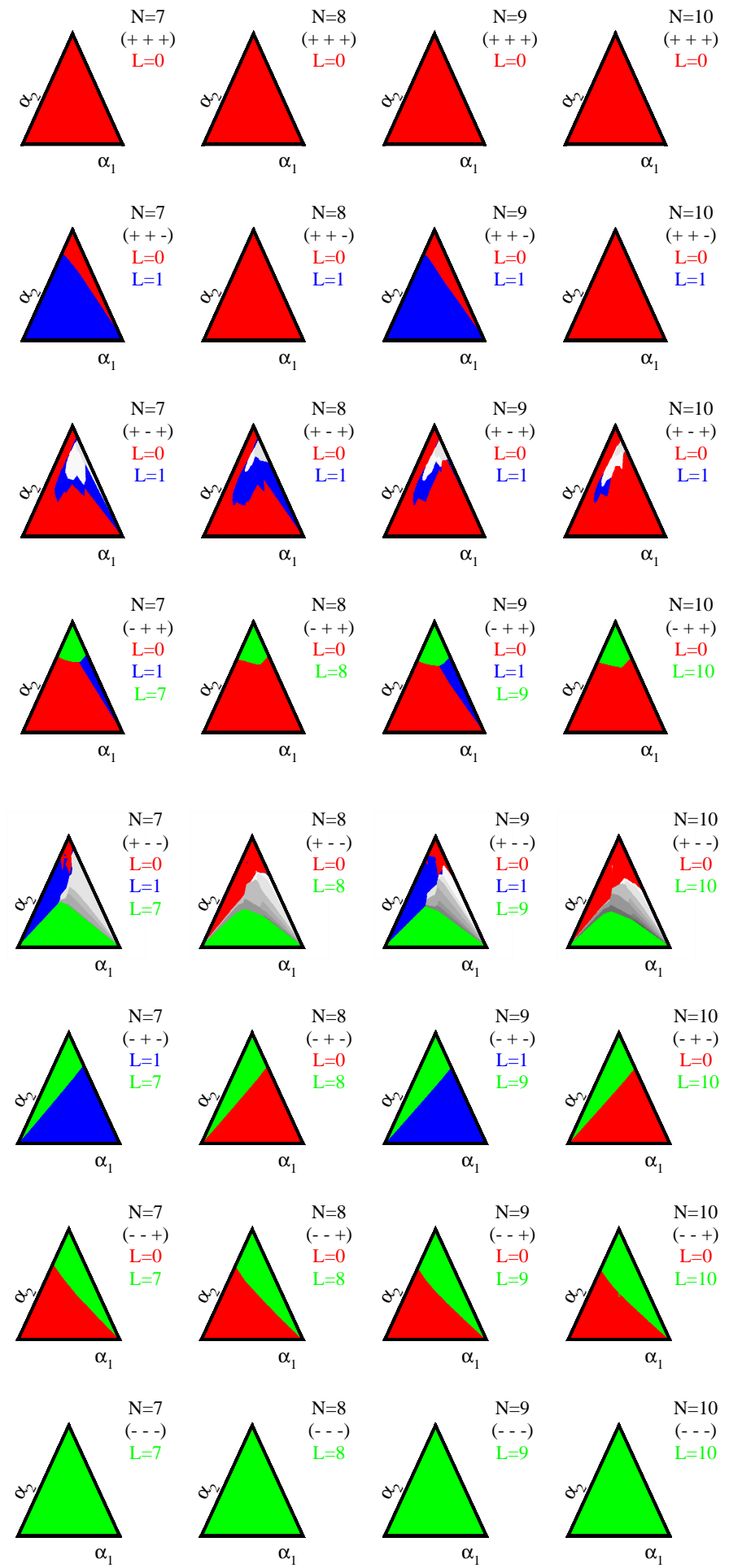

Figure 11. Angular momentum $L$ for $N=7,8,9$ and 10 bosons with the threebody vibron Hamiltonian (29), as derived from the comparison of the estimates of the lowest eigenvalues. If the angular momentum of the estimate of the eight classes of Hamiltonians $\hat{H}_{\text {vibron }}^{\varepsilon_{1}, \varepsilon_{2}, \varepsilon_{3}}\left(\alpha_{1}, \alpha_{2}\right)$ (with $\varepsilon_{i}= \pm$ ) is $L=0,1$ or $N$, the pixel at the point $\left(\alpha_{1}, \alpha_{2}\right)$ in the triangle is red, blue or green, respectively. The grey pixels represent points for which $2 \leq L \leq N-1$. Ten points have been used to compute these estimates. 

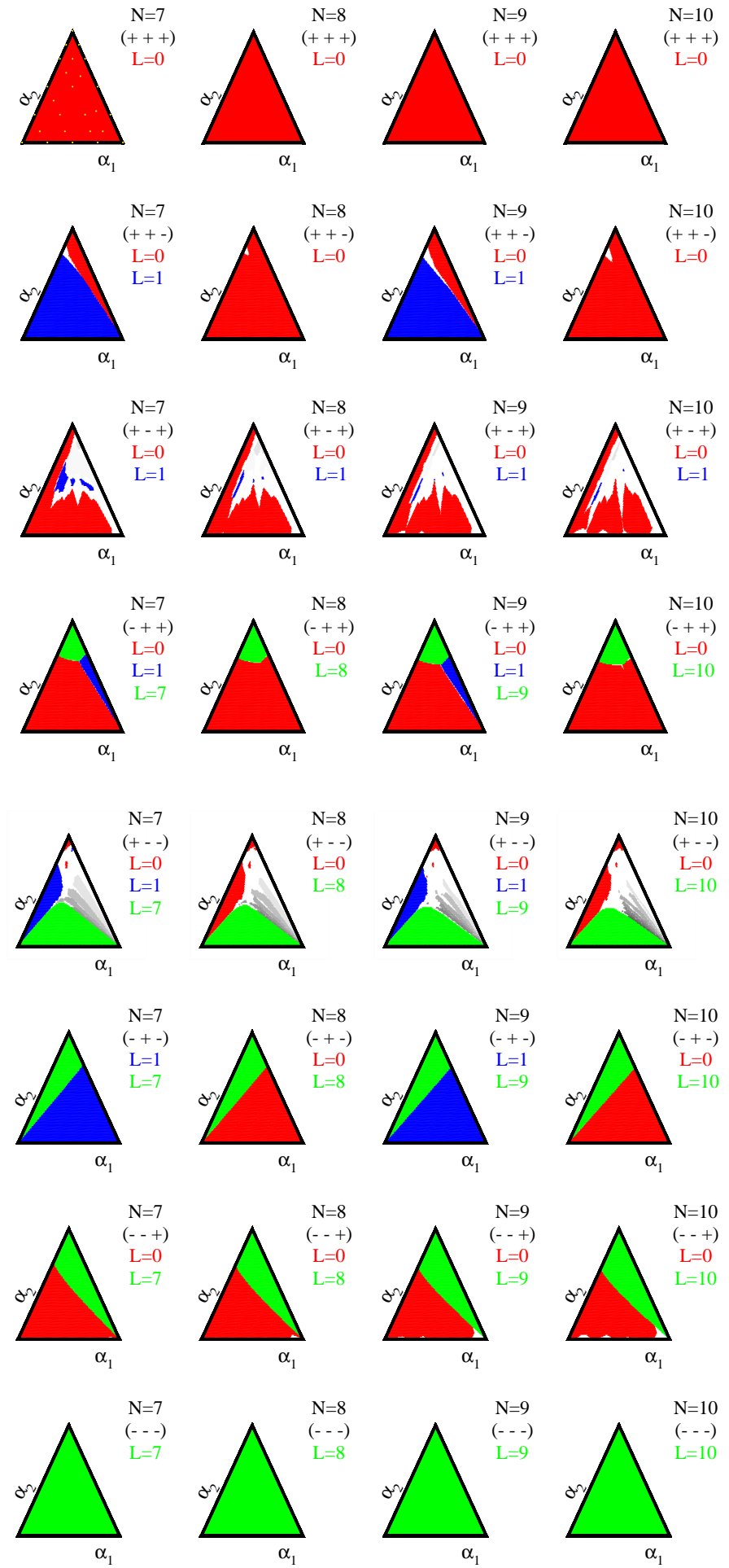

Figure 12. Ground-state angular momentum $L$ for $N=7,8,9$ and 10 bosons with the three-body vibron Hamiltonian (29), as derived from the lower (21) and upper (22) bounds of the lowest eigenvalues. If the angular momentum of the ground state of the eight classes of Hamiltonians $\hat{H}_{\text {vibron }}^{\varepsilon_{1}, \varepsilon_{2}, \varepsilon_{3}}\left(\alpha_{1}, \alpha_{2}\right)$ (with $\varepsilon_{i}= \pm$ ) is $L=0,1, N$, or $2 \leq L \leq N-1$ the pixel at the point $\left(\alpha_{1}, \alpha_{2}\right)$ in the triangle is red, blue, green, or grey respectively. In white areas no definite ground-state angular momentum could be deduced from the inequalities. The yellow dots on the first triangle indicate the twenty-six points used to derive the inequalities. 

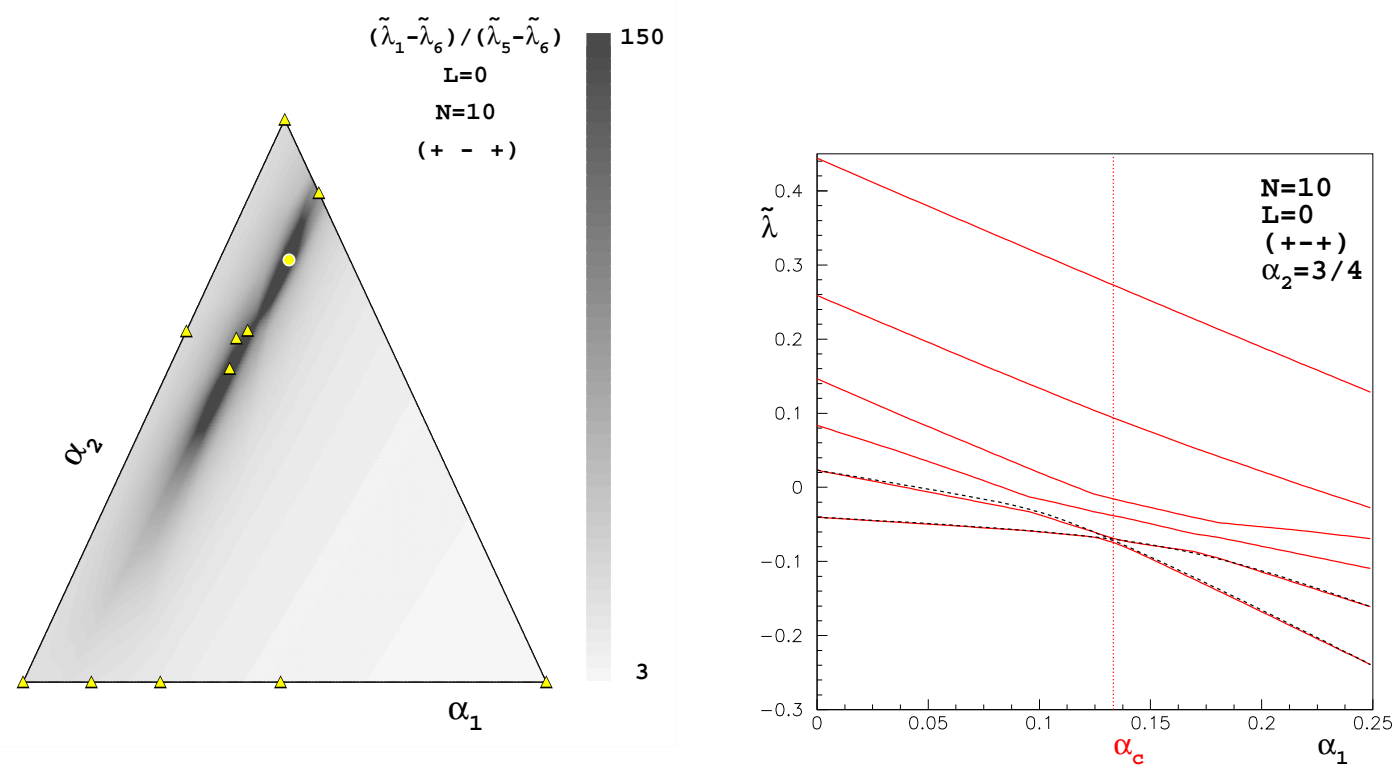

Figure 13. Properties of the exact and approximate eigenvalues $\lambda_{i}$ and $\tilde{\lambda}_{i}$ of the three-body vibron Hamiltonian (29) for $N=10$ bosons and for angular momentum $L=0$. Left panel: The ratio $\left(\tilde{\lambda}_{1}-\tilde{\lambda}_{6}\right) /\left(\tilde{\lambda}_{5}-\tilde{\lambda}_{6}\right)$ for all $\alpha_{1} \in[0,1]$ and $\alpha_{2} \in[0,1]$. Right panel: The approximate eigenvalues $\tilde{\lambda}_{i}$ (red) and the two lowest exact eigenvalues $\lambda_{5}$ and $\lambda_{6}$ (black) for $\alpha_{2}=3 / 4$ and $\alpha_{1} \in[0,1 / 4]$.

the ratio $\left(\tilde{\lambda}_{1}-\tilde{\lambda}_{6}\right) /\left(\tilde{\lambda}_{5}-\tilde{\lambda}_{6}\right)$ is plotted as a function of $\left(\alpha_{1}, \alpha_{2}\right)$, for $N=10$ bosons and for angular momentum $L=0$. The yellow triangles indicate the values of $\left(\alpha_{1}^{(v)}, \alpha_{2}^{(v)}\right)$ $(v=1, \ldots, 11 \S)$ for which the spectra are calculated. From these points one obtains approximate eigenvalues for all $\left(\alpha_{1}, \alpha_{2}\right)$, from which the ratio $\left(\tilde{\lambda}_{1}-\tilde{\lambda}_{6}\right) /\left(\tilde{\lambda}_{5}-\tilde{\lambda}_{6}\right)$ is computed. The latter quantity exhibits two maxima, one of which is indicated by the yellow dot. One suspects that level crossings may occur at these maxima. On the right panel of figure 13 are shown the approximate eigenvalues $\tilde{\lambda}_{i}$ and the two lowest exact eigenvalues $\lambda_{5}$ and $\lambda_{6}$ for $\alpha_{2}=3 / 4$ and $\alpha_{1} \in[0,1 / 4]$. For a certain critical value $\alpha_{1}=\alpha_{\mathrm{c}}$ the exact eigenvalues $\lambda_{5}$ and $\lambda_{6}$ are closest and this behaviour is correctly reproduced by the approximate eigenvalues $\tilde{\lambda}_{5}$ and $\tilde{\lambda}_{6}$. The absolute values of the components $\left|c_{j}\right|$ $(j=1, \ldots, 6)$ of the approximate eigenvectors $\left|0_{1}^{+}\right\rangle$and $\left|0_{2}^{+}\right\rangle$associated with the two lowest levels are plotted in figure 14. An abrupt shift is clearly observed at $\alpha_{1}=\alpha_{\mathrm{c}}$ for all components, indicating that a quantum phase transition is predicted to occur at the point $\left(\alpha_{1}=\alpha_{\mathrm{c}}, 3 / 4\right)$.

$\S$ Eleven points are used. The calculation starts with ten points which yield two maxima in the ratio $\left(\tilde{\lambda}_{1}-\tilde{\lambda}_{6}\right) /\left(\tilde{\lambda}_{5}-\tilde{\lambda}_{6}\right)$. Therefore, a point is added between the two maxima to improve the accuracy in the computation of the spectral projectors. 

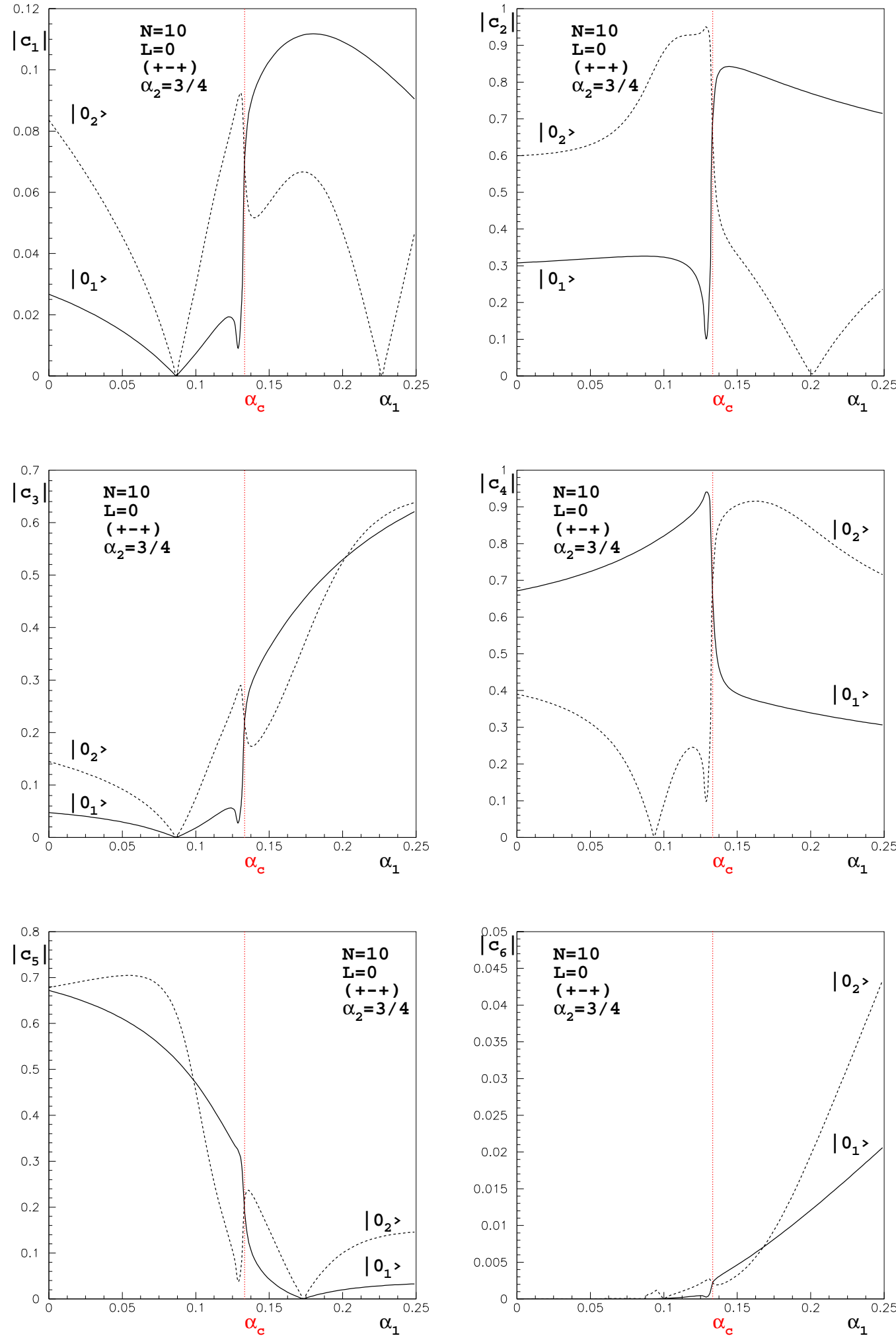

Figure 14. Absolute values of the components $\left|c_{j}\right|(j=1, \ldots, 6)$ of the approximate eigenvectors $\left|0_{1}^{+}\right\rangle$(solid) and $\left|0_{2}^{+}\right\rangle$(dashed) associated with to two lowest eigenvalues of the three-body vibron Hamiltonian (29) for $N=10$ bosons, for angular momentum $L=0$, and for $\alpha_{2}=3 / 4$ and $\alpha_{1} \in[0,1 / 4]$. 


\section{Conclusion}

In this article we have recalled several properties that can be deduced from the fact that the sums of the largest eigenvalues of Hermitian matrices are convex and we have shown how to derive inequalities from these convexity properties. We have proposed a new estimate of convex functions and have deduced from it an approximation of the eigenvalues of sums of Hermitian matrices and of their spectral projectors.

It has to be emphasized that these results must not be interpreted as just a new numerical approach to solve an eigenvalue problem, even though they can be used in that way. Rather, they are as intrinsic as the fact that eigenvalues of Hermitian matrices are real numbers and one may take advantage of them to deduce information on the spectral properties of sums of Hermitian matrices. It has also to be noted that the results presented here are different from any perturbation theory which are powerful tools [21] since convexity is a global feature and that, in some sense, they go beyond perturbation theory and are not limited to small variations.

As an illustration, these general results have been applied in the study of spectral properties of a particular quantum-mechanical model, namely the vibron model of molecules. We have derived some ground-state features of this model and, specifically, we have shown that our eigenvalue estimates provide a new way to tackle the problem of the preponderance of ground states with a certain angular momentum when random interactions between the bosons are taken. We have also studied the effect of a three-body interaction on this preponderance and shown that ground-state features are strongly affected by the rank of the interaction.

The properties of convexity considered in this paper are valid under very general conditions. The derived eigenvalue estimates can be applied to model Hamiltonians describing a wide class of time-independent quantum systems, and, consequently, many properties of such systems can be inferred from the knowledge of a limited number of spectra. We believe, therefore, that our results pave the way for applications to a variety of problems in different fields of quantum physics. Those applications might include effects of three-body or other (such as tensor) interactions on nuclear spectroscopy, ratios of excitation energies of specific levels in nuclei such as $E_{\mathrm{x}}\left(4_{1}^{+}\right) / E_{\mathrm{x}}\left(2_{1}^{+}\right)$, the existence of energy gaps in quantum systems, the values of matrix elements of transition operators or the study of quantum-phase transitional behaviour in nuclei and other mesoscopic systems. Moreover, generalizations to lower semi-bounded or compact selfadjoint operators on an infinite-dimensional Hilbert space can be carried out and should be submitted soon.

\section{Acknowledgments}

We wish to thank Marc Dupuis for his help with the figures. 


\section{References}

[1] Weyl H 1912 Math. Ann. 71441

[2] Lidskii B V 1982 Funkts. Anal. Prolozhen 16 76; Lidskii B V 1982 Functional Anal. Appl. 16139

[3] Bhatia R 2007 Perturbations Bounds for Matrix Eigenvalues (Philadelphia: SIAM, Society for Industrial and Applied Mathematics)

[4] Lewis A S 1996 Math. Oper. Res. 21576

[5] Iachello F and Levine R D 1981 Chem. Phys. Lett. 78581

[6] Hiriart-Urruty J-B and Lemaréchal C 1996 Convex Analysis and Minimization Algorithms, vol 1 (Berlin: Springer-Verlag)

[7] Texier-Picard R, Convexité et Applications (notes de cours)

[8] Hiriart-Urruty J-B and Lemaréchal C 2003 Fundamentals of Convex Analysis (Berlin: SpringerVerlag)

[9] Turner L R, 1958 NASA Technical Note NASA TN D-357

[10] Hiriart-Urruty J-B and Ye D 1995 Numer. Math. 7045

[11] Iachello F and Levine R D 1995 Algebraic Theory of Molecules (Oxford: Oxford University Press)

[12] Frank A and Van Isacker P 1994 Algebraic Methods in Molecular and Nuclear Structure Physics (New York: Wiley-Interscience)

[13] Johnson C W, Bertsch G F and Dean D J 1998 Phys. Rev. Lett. 802749

[14] Johnson C W, Bertsch G F, Dean D J and Talmi I 2000 Phys. Rev. C 61014311

[15] Bijker R, Frank A and Pittel S 1999 Phys. Rev. C 60021302

[16] Bijker R and Frank A 2000 Phys. Rev. Lett. 84420

[17] Bijker R and Frank A 2001 Phys. Rev. C 64 061303(R)

[18] Bijker R and Frank A 2002 Phys. Rev. C 65044316

[19] Vyas M and Kota V K B 2010 Ann. Phys. 325 2451; Lei Y, Zhao Y M, Yoshida N and Arima A 2011 Phys. Rev. C 83 044302; Horoi M and Zelevinsky V 2010 Phys. Rev. C 81 034306; Papenbrock T and Weidenmüller H A 2007 Rev. Mod. Phys. 79 997; Zelevinsky V and Volya A 2004 Phys. Rep. 391 311; Chau Huu-Tai P, Frank A, Smirnova N A and Van Isacker P 2002 Phys. Rev. C $66061302(\mathrm{R})$

[20] Cejnar P, Jolie J and Casten R F 2010 Rev. Mod. Phys. 822155

[21] Kato T 1976 Perturbation Theory for Linear Operators, Second Edition (Berlin: Springer-Verlag) 